\title{
Resonance Phenomena for a Class of Partial Differential Equations of Higher Order in Cylindrical Waveguides
}

\author{
P. Lesky Jr.
}

Mathematisches Institut A, Universität Stuttgart, Pfaffenwaldring 57, D-7000 Stuttgart 80, West Germany

Communicated by P. Werner

\begin{abstract}
We consider a domain $\Omega$ in $R^{n}$ of the form $\Omega=R^{l} \times \Omega^{\prime}$ with bounded $\Omega^{\prime}=R^{a-1}$. In $\Omega$ we study the Dirichlet initial and boundary value problem for the equation $\partial_{i}^{2} u+\left[\left(-\partial_{1}^{2}-\cdots-\partial_{l}^{2}\right)^{m}+\left(-\partial_{l+1}^{2}\right.\right.$ $\left.\left.-\cdots-\partial_{n}^{2}\right)^{m}\right] u=f e^{-i m t}$. We show that resonances can occur if $2 m \geqslant l$. In particular, the amplitude of $u$ may increase like $t^{\alpha}$ ( $\alpha$ rational, $0<\alpha<1$ ) or like $\ln t$ as $t \rightarrow \infty$. Furthermore, we prove that the limiting amplitude principle holds in the remaining cases.
\end{abstract}

\section{Introduction}

Let $\Omega$ be an unbounded domain in $\mathbb{R}^{n}$ with

$$
\Omega=\mathbb{R}^{l} \times \Omega^{\prime},
$$

where $\Omega^{\prime} \subset \mathbb{R}^{n-1}$ is bounded. In the following we study the solution of the initial and boundary value problem

$$
\begin{array}{ll}
\partial_{t}^{2} u+\left[\left(-\Delta_{x}\right)^{m}+\left(-\Delta_{y}\right)^{m}\right] u=f \mathrm{e}^{-\mathrm{i} \omega t} & \text { in } \Omega \times[0, \infty), \\
u=\frac{\partial u}{\partial \mathbf{n}}=\ldots=\frac{\partial^{m-1} u}{\partial \mathbf{n}^{m-1}}=0 & \text { on } \partial \Omega \times[0, \infty), \\
u(\mathbf{x}, 0)=u_{0}(\mathbf{x}), \quad \partial_{t} u(\mathbf{x}, 0)=u_{1}(\mathbf{x}) & \text { in } \Omega,
\end{array}
$$

with $\Delta_{x}:=\partial_{1}^{2}+\cdots+\partial_{l}^{2}$ and $\Delta_{y}:=\partial_{l+1}^{2}+\cdots+\partial_{n}^{2}$, $\mathbf{n}$ denotes the unit normal vector on $\partial \Omega$. For the sake of simplicity, we assume that $f, u_{0}, u_{1} \in C_{0}^{\infty}(\Omega)$ and $\partial \Omega \in C^{\infty}$. If $m=1$, then (1.2) coincides with the wave equation. We are mainly interested in the asymptotic behaviour of $u(\mathbf{x}, t)$ as $t \rightarrow \infty$.

The investigations in this paper are motivated by the results of Ramm and Werner 6 and Werner, ${ }^{12}$ who studied problem (1.2)-(1.4) in the case $m=1$ for the domains $\Omega=\mathbb{R}^{l} \times(0,1)$ and $\Omega=\mathbb{R} \times \Omega^{\prime}$ with bounded $\Omega^{\prime} \subset \mathbb{R}^{n-1}$, respectively. These configurations allow the following resonances: if $\Omega=\mathbb{R} \times \Omega^{\prime}$ and if $\omega^{2}$ is an eigenvalue of 
the Dirichlet problem

$$
-\Delta_{y} V-\lambda V=0 \text { in } \Omega^{\prime}, \quad V=0 \text { on } \partial \Omega^{\prime}
$$

for the cross-section $\Omega^{\prime}$, then

$$
u(\mathbf{x}, t)=t^{1 / 2} \mathrm{e}^{-\mathrm{i} \omega t} v(\mathbf{x})+O(1) \quad \text { as } \quad t \rightarrow \infty
$$

with a suitable $v \in C(\bar{\Omega})$. If $\Omega=\mathbb{R}^{2} \times(0,1)$ and $\omega=\pi j(j=0,1, \ldots)$, then

$$
u(\mathbf{x}, t)=\ln t \cdot \mathrm{e}^{-\mathrm{i} \omega t} v(\mathbf{x})+O(1) \quad \text { as } t \rightarrow \infty .
$$

In the case of the remaining frequencies $u(x, t)$ is bounded as $t \rightarrow \infty$ and satisfies the limiting amplitude principle

$$
u(\mathbf{x}, t)=U_{\omega}(\mathbf{x}) \mathrm{e}^{-\mathrm{i} \omega t}+o(1) \quad \text { as } t \rightarrow \infty,
$$

where $U_{\omega}$ denotes a solution of the boundary value problem

$$
\left(-\Delta_{x}-\Delta_{y}\right) U_{\omega}-\omega^{2} U_{\omega}=f \text { in } \Omega, \quad U_{\omega}=0 \text { on } \partial \Omega \text {. }
$$

The estimates (1.6)-(1.8) hold uniformly in every bounded subset of $\bar{\Omega}$. If $\Omega=\mathbb{R}^{l}$ $\times(0,1)$ with $l \geqslant 3$, then the limiting amplitude principle (1.8)-(1.9) holds for every frequency $\omega \geqslant 0$, so that no resonances occur. Hence, in the special case $m=1$, problem (1.2)-(1.4) admits resonances only if $l=1$ or $l=2$ in (1.1). The resonances are of order $t^{1 / 2}$ for $l=1$ and of order $\ln t$ for $l=2$.

In the following, we study (1.2)-(1.4) also for $m>1$ and prove that resonances are not possible if $l>2 m$. If $l \leqslant 2 m$, resonances of order $t^{\alpha}$ with rational $\alpha \in(0,1)$ or order In $t$ can occur for suitable frequencies. In particular, we obtain the following results: if $l>2 m$, then for every given $\omega \geqslant 0$ there exists a solution $U_{\omega}$ of the boundary value problem

$$
\begin{array}{ll}
{\left[\left(-\Delta_{x}\right)^{m}+\left(-\Delta_{y}\right)^{m}\right] U_{\omega}-\omega^{2} U_{\omega}=f} & \text { in } \Omega, \\
U_{\omega}=\frac{\partial U_{\omega}}{\partial \mathbf{n}}=\cdots=\frac{\partial^{m-1} U_{\omega}}{\partial \mathbf{n}^{m-1}}=0 & \text { on } \partial \Omega,
\end{array}
$$

such that (1.8) holds uniformly in every bounded subset of $\bar{\Omega}$ (limiting amplitude principle). $U_{\omega}$ can be uniquely characterized by a suitable radiation condition, which can be considered as a generalization of a uniqueness condition introduced by Sveshnikov ${ }^{7}$ in the case $m=1$. Furthermore, (1.8) and (1.10) remain valid if $l \leqslant 2 m$ and if $\omega^{2}$ does not coincide with one of the eigenvalues $\lambda_{1}, \lambda_{2}, \ldots$ of the Dirichlet problem

$$
\begin{array}{ll}
\left(-\Delta_{y}\right)^{m} V-\lambda V=0 & \text { in } \Omega^{\prime}, \\
V=\frac{\partial V}{\partial n^{\prime}}=\cdots=\frac{\partial^{m-1} V}{\partial n^{\prime m-1}}=0 & \text { on } \partial \Omega^{\prime}
\end{array}
$$

for the cross-section $\Omega^{\prime}\left(n^{\prime}:=\right.$ unit normal vector on $\left.\partial \Omega^{\prime}\right)$. However, if $l \leqslant 2 m$ and $\omega^{2}=\lambda_{j}$, then the following resonance phenomenon can be observed: if $l$ is odd, then the estimate

$$
u(\mathbf{x}, t)=\sum_{s=0}^{m-1 l+1) / 2}=E_{s} t^{1-\frac{1+2 s}{2 m}} \mathrm{e}^{-\mathrm{i} \omega t} p_{s}^{(j)}(\mathbf{x})+U(\mathbf{x}) \mathrm{e}^{-\mathrm{i} \omega t}+o(1) \text { as } t \rightarrow \infty
$$

holds; here $U$ is a solution of (1.10), $E_{s}$ are suitable constants and 


$$
p_{s}^{(j)}(\mathbf{x})=p_{s}^{(j)}(x, y):=\sum_{k=1}^{k(j)} V_{j k}(y) \int_{\Omega} f\left(x^{\prime}, y^{\prime}\right) V_{j k}\left(y^{\prime}\right)\left|x-x^{\prime}\right|^{2 s} d\left(x^{\prime}, y^{\prime}\right),
$$

where $x:=\left(x_{1}, \ldots, x_{1}\right), y:=\left(x_{1+1}, \ldots, x_{n}\right)$ and $V_{j k}(k=1, \ldots \kappa(j))$ denotes an orthonormal basis of the eigenspace $E_{j}$ belonging to the eigenvalue $\lambda_{j}$ of (1.11). For even $l(1.12)$ has to be replaced by

$$
\begin{aligned}
u(x, t)= & \sum_{s=0}^{m-1-1 / 2} E_{s} t^{1-\frac{1+2 s}{2 m}} e^{-\mathrm{i} \omega t} p_{s}^{(j)}(\mathbf{x})+E^{*} \ln t \cdot \mathrm{e}^{-\mathrm{i} \omega t} p_{m-l / 2}^{(j)}(\mathbf{x}) \\
& +U(\mathbf{x}) \mathrm{e}^{-\mathrm{i} \omega t}+o(1) \quad \text { as } t \rightarrow \infty .
\end{aligned}
$$

Both estimates hold uniformly in every bounded subset of $\bar{\Omega}$. The precise values of $E_{s}$ and $E^{*}$ will be given in Section 6.

The analysis of this paper is based on the spectral theory for unbounded self-adjoint operators. In Section 2, we extend the differential operator $\left(-\Delta_{x}\right)^{m}+\left(-\Delta_{y}\right)^{m}$ to a selfadjoint operator $A$ in the Hilbert space $L_{2}(\Omega)$ with respect to Dirichlet's boundary condition (1.3). Applying the functional calculus for unbounded self-adjoint operators, we obtain a spectral integral representation for the solution $u$ of (1.2)-(1.4). Sections 3 and 4 are deveoted to the study of the resolvent of $A$ and the construction of the spectral family $\left\{P_{\lambda}\right\}$ of $A$. The explicit form of $\left\{P_{\lambda}\right\}$ is used in Sections 5 and 6 to obtain the above asymptotic for $u(x, t)$ as $t \rightarrow \infty$.

The results of this paper are contained in the author's thesis, ${ }^{3}$ to which we refer for a more detailed presentation of some of the proofs.

\section{The spectral integral representation of the solution}

In order to extend the operator $\left(-\Delta_{x}\right)^{m}+\left(-\Delta_{y}\right)^{m}$ to a self-adjoint operator with respect to the Dirichlet condition (1.3), we set

$$
\left.\begin{array}{rl}
D(A) & :=\left\{U \in \stackrel{\circ}{H}_{m}(\Omega):\left[\left(-\Delta_{x}\right)^{m}+\left(-\Delta_{y}\right)^{m}\right] U \in L_{2}(\Omega)\right\}, \\
A U & :=\left[\left(-\Delta_{x}\right)^{m}+\left(-\Delta_{y}\right)^{m}\right] U \text { for } U \in D(A) ;
\end{array}\right\}
$$

here the differential operators $\left(-\Delta_{x}\right)^{m}$ and $\left(-\Delta_{y}\right)^{m}$ are interpreted in the sense of distribution theory and $\stackrel{\circ}{H}_{m}(\Omega)$ denotes the closure of $C_{0}^{\infty}(\Omega)$ in the Sobolev space $H_{m}(\Omega)$ (compare, for example, Reference 8 ). We prove:

Lemma 2.1. The operator $A$ defined by (2.1) is positive and self-adjoint in the Hilbert space $L_{2}(\Omega)$.

Proof. Consider the bilinear form

$$
B(U, V):= \begin{cases}\left(\Delta_{x}^{m / 2} U, \Delta_{x}^{m / 2} V\right)+\left(\Delta_{y}^{m / 2} U, \Delta_{y}^{m / 2} V\right) & (m \text { even }), \\ \left(\nabla_{x} \Delta_{x}^{(m-1) / 2} U, \nabla_{x} \Delta_{x}^{(m-1) / 2} V+\right. & \\ \left(\nabla_{y} \Delta_{y}^{(m-1) / 2} U, \nabla_{y} \Delta_{y}^{(m-1) / 2} V\right) & (m \text { odd }),\end{cases}
$$

for $U, V \in \stackrel{\circ}{H}_{m}(\Omega)$ with

$$
\left(\nabla_{x} U, \nabla_{x} V\right):=\sum_{i=1}^{l}\left(\partial_{i} U, \partial_{i} V\right), \quad\left(\nabla_{y} U, \nabla_{y} V\right):=\sum_{i=l+1}^{n}\left(\partial_{i} U, \partial_{i} V\right)
$$


$\left(():,=\right.$ inner product in $\left.L_{2}(\Omega)\right)$. Assume that $U \in D(A)$ and $\varphi \in C_{0}^{\infty}(\Omega)$. Then

$$
\begin{aligned}
(A U, \varphi) & =\left(\left[\left(-\Delta_{x}\right)^{m}+\left(-\Delta_{y}\right)^{m}\right] U, \varphi\right)=\left[\left(-\Delta_{x}\right)^{m} U+\left(-\Delta_{y}\right)^{m} U\right](\bar{\varphi}) \\
& =\sum_{i=1}^{l}\left[\partial_{i}\left(-\Delta_{x}\right)^{m-1} U\right]\left(\partial_{i} \bar{\varphi}\right)+\sum_{i=1+1}^{n}\left[\partial_{i}\left(-\Delta_{y}\right)^{m-1} U\right]\left(\partial_{i} \bar{\varphi}\right),
\end{aligned}
$$

where $F(\varphi)$ denotes the application of the distribution $F$ to the test function $\varphi$. Repeating this step $m$ times, we obtain $(A, U, \varphi)=B(U, \varphi)$, and hence

$$
B(U, V)=(A U, V) \quad \text { for } U, V \in D(A),
$$

since $C_{0}^{\infty}(\Omega)$ is dense in $D(A)$. This implies that $A$ is symmetric and positive.

Now we show that $A$ is elliptic. Substituting $x_{i}$ for $\partial_{i} U$ and $\partial_{i} V$ in (2.2), we obtain for odd $m$

$$
\begin{aligned}
B(\mathbf{x}, \mathbf{x})= & \sum_{j=1}^{l}\left(x_{j}\left(\sum_{i=1}^{l} x_{i}^{2}\right)^{(m-1) / 2} \cdot x_{j}\left(\sum_{i=1}^{l} x_{i}^{2}\right)^{(m-1) / 2}\right) \\
& +\sum_{j=l+1}^{n}\left(x_{j}\left(\sum_{i=l+1}^{n} x_{i}^{2}\right)^{(m-1) / 2} \cdot x_{j}\left(\sum_{i=l+1}^{n} x_{i}^{2}\right)^{(m-1) / 2}\right),
\end{aligned}
$$

and hence

$$
B(\mathbf{x}, \mathbf{x})=\left(\sum_{i=1}^{l} x_{i}^{2}\right)^{m}+\left(\sum_{i=l+1}^{n} x_{i}^{2}\right)^{m}
$$

By (2.2) this identity holds also for even $m$. Hölder's inequality implies

$$
\left|\sum_{j=1}^{2} a_{j} \cdot 1\right| \leqslant\left(\sum_{j=1}^{2}\left|a_{j}\right|^{m}\right)^{1 / m}\left(\sum_{j=1}^{2} 1\right)^{(m-1) / m},
$$

so that

$$
B(\mathbf{x}, \mathbf{x}) \geqslant \frac{1}{2^{m-1}}|\mathbf{x}|^{2 m} \quad \text { for } \mathbf{x} \in \mathbb{R}^{n} .
$$

Thus $A$ is uniformly strongly elliptic. In particular, Gårdings inequality

$$
B(U, U) \geqslant c_{1}\|U\|_{m}^{2}-c_{2}\|U\|^{2} \quad \text { for } U \in \mathscr{H}_{m}(\Omega)
$$

holds with suitable $c_{1}, c_{2}>0$. This implies by a familiar argument that $A+c_{2}$ is a bijective linear mapping from $D(A)$ onto $L_{2}(\Omega)$. Hence $A$ is self-adjoint. This concludes the proof of Lemma 2.1.

We consider the following weak formulation of problem (1.2)-(1.4): find $U \in C^{2}\left([0, \infty), L_{2}(\Omega)\right)$ such that

$$
\begin{aligned}
& U^{\prime \prime}(t)+A U(t)=f \mathrm{e}^{-\mathrm{i} \omega t} \quad \text { for } t \geqslant 0, \\
& U(0)=u_{0}, U^{\prime}(0)=u_{1} ;
\end{aligned}
$$

here $U^{\prime}(t)$ denotes the derivative of the mapping $U:[0, \infty) \rightarrow L_{2}(\Omega)$ with respect to the $L_{2}$ norm. By using (2.3) it can be shown that $U$ is uniquely determined (compare the proof of Lemma 7.1 in Reference 9). Let $\left\{\boldsymbol{P}_{\lambda}\right\}$ be the (left continuous) spectral family of 
$A$. Note that $P_{\lambda}=0$ for $\lambda \leqslant 0$, since $A$ is positive. Set

$$
U(t):=\int_{0}^{\infty} \cos \sqrt{\lambda} t \mathrm{~d}\left(P_{\lambda} u_{0}\right)+\int_{0}^{\infty} \frac{\sin \sqrt{\lambda} t}{\sqrt{\lambda}} \mathrm{d}\left(P_{\lambda} u_{1}\right)+\int_{0}^{\infty} \psi(\lambda, t) \mathrm{d}\left(P_{\lambda} f\right),
$$

where $\psi(\lambda, t)$ is the solution of the initial value problem

$$
\left(\partial_{t}^{2}+\lambda\right) \psi(\lambda, t)=\mathrm{e}^{-\mathrm{i} \omega t} \text { for } t \geqslant 0, \psi(\lambda, 0)=\partial_{t} \psi(\lambda, 0)=0,
$$

given by

$$
\psi(\lambda, t)= \begin{cases}\frac{1}{\lambda-\omega^{2}}\left(\mathrm{e}^{-\mathrm{i} \omega t}-\cos \sqrt{\lambda} t+\frac{\mathrm{i} \omega}{\sqrt{\lambda}} \sin \sqrt{\lambda} t\right) & \text { if } \lambda \neq \omega^{2} \\ \frac{\mathrm{i}}{2 \omega}\left(t \mathrm{e}^{-\mathrm{i} \omega t}-\frac{1}{\omega} \sin \omega t\right) & \text { if } \lambda=\omega^{2} .\end{cases}
$$

The functional calculus for unbounded self-adjoint operators shows that $U$ is a solution of (2.7). Since $f, u_{0}, u_{1} \in C_{0}^{\infty}(\Omega) \subset D\left(A^{r}\right)$ for every $r \in \mathbb{N}$, it follows by the elliptic regularity theory that $(1.2)-(1.4)$ has a classical solution $u(x, t)$ belonging to $C^{\infty}(\bar{\Omega} \times[0, \infty))$, which is related to $U(t)$ by

$$
U(t) \varphi=\int_{\Omega} u(\mathbf{x}, t) \varphi(\mathbf{x}) \mathrm{d} \mathbf{x} \quad \text { for every } \varphi \in C_{0}^{\infty}(\Omega)
$$

(compare, for example, the discussion in Reference 10 in a related situation). On the other hand, every solution $u$ of (1.2)-(1.4) having the property

$$
u(., t) \in H_{m}(\Omega) \quad \text { for every } t \geqslant 0
$$

defines a solution $u$ of $(2.7)$ by (2.11). Thus we obtain:

Lemma 2.2. Problem (1.2)-(1.4) has one and only one solution $u$ with the property (2.12); $u$ is given by (2.8) and (2.10).

\section{The resolvent}

In order to estimate the asymptotic behaviour of the solution $u$ given by (2.8) and (2.10), we have to study the behaviour of the resolvent $R_{z}=(A-z I)^{-1}$ of $A$ near the real axis. The computation of $R_{z} f$ for $z \in \mathbb{C} \backslash[0, \infty)$ and $f \in C_{0}^{\infty}(\Omega)$ leads to the classical problem

$$
\begin{array}{ll}
{\left[\left(-\Delta_{x}\right)^{m}+\left(-\Delta_{y}\right)^{m}-z\right] U_{z}=f} & \text { in } \Omega, \\
U_{z}=\frac{\partial U_{z}}{\partial \mathbf{n}}=\cdots=\frac{\partial^{m-1} U_{z}}{\partial \mathbf{n}^{m-1}}=0 & \text { on } \partial \Omega
\end{array}
$$

We construct a solution $U_{z}$ of $(3.1)$ by setting

$$
U_{z}(\mathbf{x})=U_{z}(x, y)=\sum_{j=1}^{\infty} \sum_{k=1}^{\kappa(j)} u_{j k}(x ; z) V_{j k}(y)
$$

where $V_{j k}(k=1, \ldots, \kappa(j))$ denotes as in (1.13) an orthonormal basis of the eigenspace $E_{j}$ for the eigenvalue $\lambda_{j}$ of (1.11). We assume that the eigenvalues are ordered increasingly: $0<\lambda_{1}<\lambda_{2}<\ldots$. Note that $V_{i k} \in C^{\infty}\left(\bar{\Omega}^{\prime}\right)$ by the elliptic regularity 
theory, since $\partial \Omega^{\prime} \in C^{\infty}$. Using $\left(-\Delta_{y}\right)^{m} V_{j k}=\lambda_{j} V_{j k}$, we obtain formally

$$
\left[\left(-\Delta_{x}\right)^{m}+\left(-\Delta_{y}\right)^{m}-z\right] U_{z}(\mathbf{x})=\sum_{j=1}^{\infty} \sum_{k=1}^{x(j)}\left[\left(-\Delta_{x}\right)^{m}+\lambda_{j}-z\right] u_{j k}(x ; z) V_{j k}(y) \text {. }
$$

The expansion theorem for the interior boundary value problem (1.11) yields

$$
f(\mathbf{x})=f(x, y)=\sum_{j=1}^{\infty} \sum_{k=1}^{x(j)} f_{j k}(x) V_{j k}(y)
$$

with

$$
f_{j k}(x)=\int_{\Omega^{\prime}} f(x, y) \bar{V}_{j k}(y) \mathrm{d} y .
$$

These considerations suggest determining the coefficients in (3.2) such that

$$
\left[\left(-\Delta_{x}\right)^{m}+\lambda_{j}-z\right] u_{j k}=f_{j k} \quad \text { in } \mathbb{R}^{l} \text {. }
$$

In order to compute $u_{j k}$, we extend $\left(-\Delta_{x}\right)^{m}$ to a self-adjoint operator $L(m)$ in $L_{2}\left(\mathbb{R}^{l}\right)$ by setting

$$
\left.\begin{array}{rl}
D(L(m)) & :=\left\{W \in H_{m}\left(\mathbb{R}^{l}\right):\left(-\Delta_{x}\right)^{m} W \in L_{2}\left(\mathbb{R}^{l}\right)\right\}, \\
L(m) W & :=\left(-\Delta_{x}\right)^{m} W \quad \text { for } W \in D(L(m)) .
\end{array}\right\}
$$

Since $L(m)$ is uniformly strongly elliptic, the elliptic regularity theory implies $L(m)=L(1)^{m}$. Hence, the uniquely determined solution $u_{j k}$ of (3.6) belonging to $D(L(m))$ coincides with the solution of

$$
\left[L(1)^{m}+\lambda_{j}-z\right] u_{j k}=f_{j k} .
$$

Let $\left\{P_{\lambda}^{(1)}\right\}$ denote the spectral family of $L(1)$. Then $u_{j k}$ is given by

$$
u_{j k}=\int_{0}^{\infty} \frac{1}{\lambda^{m}+\lambda_{j}-z} \mathrm{~d}\left(P_{\lambda}^{(1)} f_{j k}\right)
$$

We set $\mu_{s}:=\left|\lambda_{j}-z\right|^{1 / m} \mathrm{e}^{\mathrm{i}\left(\arg \left(z-\lambda_{j}\right)+2 \pi s\right) / m}(s=0, \ldots, m-1)$ and obtain

$$
u_{j k}=\int_{0}^{\infty}\left\{\sum_{s=0}^{m-1} \frac{1}{m \mu_{s}^{m-1}\left(\lambda-\mu_{s}\right)}\right\} \mathrm{d}\left(P_{\lambda}^{(1)} f_{j k}\right)=: \sum_{s=0}^{m-1} \frac{1}{m \mu_{s}^{m-1}} w_{s} .
$$

Here $w_{s}$ is the uniquely determined solution of $\left(-\Delta_{x}-\mu_{s}\right) w_{s}=f_{j k}$ in $\mathbb{P}^{l}$ belonging to $D(L(1)) ; w_{s}$ has the explicit form

$$
w_{s}(x ; z)=\frac{\mathrm{i}\left|\lambda_{j}-z\right|^{\sigma / 2 m}}{4(2 \pi)^{\sigma}} \mathrm{e}^{\mathrm{i} \sigma \varphi_{s}(z ; j)} \int_{\mathbf{R}^{\prime}\left|x-x^{\prime}\right|^{\sigma}} \frac{f_{j \mathrm{k}}\left(x^{\prime}\right)}{\mid(1)}\left(\left|x-x^{\prime}\right|\left|\lambda_{j}-z\right|^{1 / 2 m} \mathrm{e}^{\mathrm{i} \varphi_{s}(z ; j) / 2}\right) \mathrm{d} x^{\prime},
$$

where $\sigma:=(l / 2)-1, H_{\sigma}^{(1)}$ denotes Hankel's function,

$$
H_{\sigma}^{(1)}(\zeta)=J_{\sigma}(\zeta)+\mathrm{i} N_{\sigma}(\zeta) \quad \text { for } \zeta \in \mathbb{C}
$$

and

$$
\varphi_{s}(z ; j):=\frac{\arg \left(z-\lambda_{j}\right)+2 \pi s}{m} \quad(s=0, \ldots, m-1)
$$


for $0<\arg \left(z-\lambda_{j}\right)<2 \pi$. Thus we have

$$
\begin{aligned}
u_{j k}(x ; z)= & \frac{\mathrm{i}\left|\lambda_{j}-z\right|^{\frac{\alpha+2}{2 m}}-1}{4 m(2 \pi)^{\sigma}} \sum_{s=0}^{m-1} \mathrm{e}^{\mathrm{i} \varphi_{s}(z ; j)[(\sigma / 2)+1-m]} \\
& \int_{\mathbb{R}^{l}} \frac{f_{j k}\left(x^{\prime}\right)}{\left|x-x^{\prime}\right|^{\sigma}} H_{\sigma}^{(1)}\left(\left|x-x^{\prime}\right|\left|\lambda_{j}-z\right|^{1 / 2 m} \mathrm{e}^{\mathrm{i} \varphi_{s}(z ; j) / 2}\right) \mathrm{d} x^{\prime} .
\end{aligned}
$$

In order to proof the convergence of the Fourier series (3.2), we start from

$$
L(m) u_{j k}+\left(\lambda_{j}-2\right) u_{j k}=f_{j k}
$$

(compare (3.6) and (3.7)). It follows that

$$
\left(L(m) u_{j k}, u_{j k}\right)_{\mathbb{R}^{\prime}}+\left(\lambda_{j}-z\right)\left\|u_{j k}\right\|_{\mathbb{R}^{\prime}}^{2}=\left(f_{j k}, u_{j k}\right)_{\mathbb{R}^{\prime}}
$$

$\left((\cdot, \cdot)_{\mathbb{R}^{\prime}}:=\right.$ inner product in $L_{2}\left(\mathbb{R}^{l}\right),\|\cdot\|_{\mathbb{R}^{l}}:=$ norm in $\left.L_{2}\left(\mathbb{R}^{l}\right)\right)$. Since $L(m)$ is self-adjoint and positive, we obtain

$$
\begin{array}{r}
\operatorname{Re}\left(\dot{\lambda}_{j}-z\right)\left\|u_{j k}\right\|_{\mathbf{R}^{\prime}}^{2} \leqslant \operatorname{Re}\left(f_{j k}, u_{j k}\right)_{\mathbb{R}^{\prime}}, \\
-\operatorname{Im} z\left\|u_{j k}\right\|_{\mathbf{R}^{\prime}}^{2}=\operatorname{Im}\left(f_{j k}, u_{j k}\right)_{\mathbb{R}^{\prime}},
\end{array}
$$

and by the Cauchy-Schwarz inequality

$$
\left\|u_{j k}\right\| \mathbb{R}^{i} \leqslant c_{j}(z)\left\|\int_{j k}\right\|_{\mathbb{R}^{i}}
$$

where

$$
c_{j}(z):= \begin{cases}\min \left\{\frac{1}{|\operatorname{Im} z|}, \frac{1}{\operatorname{Re}\left(\lambda_{j}-z\right)}\right\} & \text { if } \operatorname{Re} z<\lambda_{j}, \\ \frac{1}{|\operatorname{Im} z|} & \text { if } \operatorname{Re} z \geqslant \lambda_{j} .\end{cases}
$$

The elliptic regularity theory and (3.12) imply $D^{p} u_{j k} \in D(L(m))$ and

$$
L(m) D^{p} u_{j k}+\left(\lambda_{j}-z\right) D^{p} u_{j k}=D^{p} f_{j k}
$$

for every multi-index $p \in \mathbb{N}_{0}^{l}$, since $f_{j k} \in C_{0}^{\infty}\left(\mathbb{R}^{l}\right)$. Hence, the above considerations yield

$$
\left\|u_{j k}\right\|_{s, \mathbb{R}^{\prime}} \leqslant c_{j}(z)\left\|f_{j k}\right\|_{\mathrm{s}, \mathbb{R}^{\prime}}
$$

for every $s \in \mathbb{N}_{0}\left(\|\cdot\|_{s, \mathbb{R}^{\prime}}:=\right.$ norm in $\left.H_{s}\left(\mathbb{R}^{l}\right)\right)$. Consider

$$
h_{z}(\mathbf{x}):=\sum_{j=j_{1}}^{j} \sum_{k=1}^{x(j)} u_{j k}(x ; z) V_{j k}(y)
$$

with $j_{1}, j_{2} \in \mathbb{N}, j_{2}>j_{1}$. Let $d_{j}>0$ and $i_{j} \in \mathbb{N}$ denote suitable constants. Sobolev's inequality implies

$$
\left|h_{z}(\mathbf{x})\right|^{2}=\left|h_{z}(x, y)\right|^{2} \leqslant d_{1}\left\|h_{z}(x, .)\right\|_{i_{1}, \Omega^{\prime}}^{2}
$$

for every fixed $x \in \mathbb{R}^{\prime}$. Since $\Delta_{y}^{s-1} h_{z}(x,.) \in L_{2}\left(\Omega^{\prime}\right)$ for every $s \in \mathbb{N}$, it follows from the elliptic regularity theory that

$$
\left\|\Delta_{y}^{s-1} h_{z}(x, .)\right\|_{2 m, \Omega^{\prime}}^{2} \leqslant d_{2}\left(\left\|\Delta_{y}^{s-1} h_{z}(x, .)\right\|_{\Omega^{\prime}}^{2}+\left\|\Delta_{y}^{s} h_{z}(x, .)\right\|_{\Omega^{\prime}}^{2}\right) .
$$


Using this inequality, we obtain by the same argument

$$
\left\|\Delta_{y}^{s-2} h_{z}(x, .)\right\|_{4 m, \Omega^{\prime}}^{2} \leqslant d_{2}\left\|\Delta_{y}^{s-2} h_{z}(x, .)\right\|_{\Omega^{\prime}}^{2}+d_{2}^{2}\left(\left\|\Delta_{y}^{s-1} h_{z}(x, .)\right\|_{\Omega^{\prime}}^{2}+\left\|\Delta_{y}^{s} h_{z}(x, .)\right\|_{\Omega^{\prime}}^{2}\right) \text {. }
$$

Continuing in this way, we conclude that

$$
\begin{aligned}
\left\|h_{z}(x, .)\right\|_{2 s m, \Omega^{\prime}}^{2} & \leqslant d_{3} \sum_{r=0}^{s}\left\|\Delta_{y}^{r} h_{z}(x, .)\right\|_{\Omega^{\prime}}^{2} \\
& =d_{3} \sum_{r=0}^{s} \sum_{j=j_{1}}^{j_{2}} \sum_{k=1}^{x(j)} \lambda_{j}^{2 r}\left|u_{j k}(x ; z)\right|^{2} .
\end{aligned}
$$

Since

$$
\lambda_{j}^{2 r}=\lambda_{1}^{2 r}\left(\frac{\lambda_{j}}{\lambda_{1}}\right)^{2 r} \leqslant \lambda_{1}^{2 r}\left(\frac{\lambda_{j}}{\lambda_{1}}\right)^{2 s}=\frac{1}{\lambda_{1}^{2 s-2 r}} \lambda_{j}^{2 s}
$$

we have

$$
\left\|h_{z}(x, .)\right\|_{2 s m, \Omega^{\prime}} \leqslant d_{4} \sum_{j=j_{1}}^{j z} \sum_{k=1}^{x(j)} \lambda_{j}^{2 s}\left|u_{j k}(x ; z)\right|^{2} .
$$

Combining (3.21), (3.19), Sobolev's inequality and (3.17), we obtain

$$
\begin{aligned}
\left|h_{z}(\mathbf{x})\right|^{2} & \leqslant d_{5} \sum_{j=j_{1}}^{j_{2}} \sum_{k=1}^{\kappa(j)} \lambda_{j}^{2 i_{2}}\left|u_{j k}(x ; z)\right|^{2} \\
& \leqslant d_{6} \sum_{j=j_{1}}^{j_{2}} \sum_{k=1}^{\kappa(j)} \lambda_{j}^{2 i_{2}}\left\|u_{j k}(x ; z)\right\|_{i_{3}, \mathbb{R}^{\prime}} \\
& \leqslant d_{6} c_{j_{1}}(z)^{2} \sum_{j=j_{1}}^{j_{2}} \sum_{k=1}^{\kappa(j)} \lambda_{j}^{2 i_{2}}\left\|f_{j k}\right\|_{i_{3}, \mathbb{R}^{\prime}}^{2},
\end{aligned}
$$

since $c_{j_{1}}(z) \geqslant c_{j}(z)$ for $j>j_{1}$. On the other hand, by applying Bessel's inequality to the Fourier expansion (3.4), we conclude that

$$
\sum_{j=1}^{N} \sum_{k=1}^{x(j)} \lambda_{j}^{2 i_{2}}\left|D_{x}^{p} f_{j k}(x)\right|^{2} \leqslant\left\|\Delta_{y}^{i_{2}} D_{x}^{p} f(x, .)\right\|_{\Omega^{\prime}}^{2}
$$

for every $p \in \mathbb{N}_{0}^{l}$ and $N \in \mathbb{N}\left(D_{x}^{p}:=\partial_{1}^{p_{1}}, \ldots, \partial_{l}^{p^{l}}\right)$. In particular, we have

$$
\sum_{j=1}^{\infty} \sum_{k=1}^{\kappa(j)} \lambda_{j}^{2 i_{2}}\left\|f_{j k}\right\|_{i_{3}, \mathbb{R}^{i}}^{2} \leqslant \sum_{|p| \leq i_{3}}\left\|\Delta_{y}^{i_{2}} D_{x}^{p} f\right\|_{\Omega}^{2}<\infty
$$

Thus (3.18), (3.22) and (3.28) together with (3.15) imply that the Fourier series (3.2) of $U_{z}$ converges uniformly with respect to $\mathrm{x} \in \bar{\Omega}$ and $z \in K$, if $K$ is an arbitrary compact subset of $\mathbb{C} \backslash\left[\lambda_{1}, \infty\right)$. It follows in the same way that every termwise derivative of (3.2) converges uniformly with respect to $x \in \bar{\Omega}$ and $z \in K$. Thus $U_{z}$ is a solution of the differential equation in (3.1). Furthermore, we have

$$
\frac{\partial^{s} U_{z}}{\partial \mathbf{n}^{s}}(\mathbf{x})=\sum_{j=1}^{\infty} \sum_{k=1}^{k(j)} \frac{\partial^{s}}{\partial \mathbf{n}^{s}}\left[u_{j k}(x ; z) V_{j k}(y)\right]=\sum_{j=1}^{\infty} \sum_{k=1}^{k(j)} u_{j k}(x ; z) \frac{\partial^{s}}{\partial n^{1 s}} V_{j k}(y)=0
$$

for every $\mathbf{x}=(x, y) \in \partial \Omega$ and $s=0, \ldots, m-1$, since $\mathbf{n}=\left(n_{x}, n_{y}\right)$ with $n_{x}=(0, \ldots, 0)$ and $n_{y}=n^{\prime}$, and since each $V_{j k}$ is an eigenfunction of (1.11). Hence $U_{z}$ is a solution of (3.1). 
The same argument as above yields that for $p \in \mathbb{N}_{0}^{l}, q \in \mathbb{N}_{0}^{n-1}$

$$
\begin{aligned}
\left\|D_{x}^{p} D_{y}^{q} h_{z}\right\|^{2} & =\int_{\mathbf{R}^{\prime}}\left\{\int_{\Omega^{\prime}}\left|D_{x}^{p} D_{y}^{q} h_{z}(x, y)\right|^{2} \mathrm{~d} y\right\} \mathrm{d} x \\
& \leqslant \int_{R^{\prime}}\left\|D_{x}^{p} h_{z}(x, .)\right\|_{|q|, \Omega^{\prime}}^{2} \mathrm{~d} x \\
& \leqslant d_{7} \int_{R^{\prime}}\left\{\sum_{j=j_{1}} \sum_{k=1}^{j_{2}} \lambda_{j}^{x(j)} \lambda_{j}^{2 i_{4}}\left|D_{x}^{p} u_{j k}(x ; z)\right|^{2}\right\} \mathrm{d} x \\
& =d_{7} \sum_{j=j_{1}}^{j_{2}} \sum_{k=1}^{x(j)} \lambda_{j}^{2 i_{4}}\left\|D_{x}^{p} u_{j k}(. ; z)\right\|_{\mathbf{R}^{l}}^{2} \\
& \leqslant d_{7} c_{j_{1}}(z)^{2} \sum_{j=j_{1}}^{j_{2}} \sum_{k=1}^{x(j)} \lambda_{j}^{2 i_{4}}\left\|f_{j k}\right\|_{\left\{p \mid, R^{2}\right.}^{2} \\
& \leqslant d_{7} c_{1}(z)^{2}\left\|\Delta_{y}^{i_{4}} f\right\|_{|p|, R^{\prime \cdot}}^{2}
\end{aligned}
$$

This shows that $U_{z} \in H_{s}(\Omega)$ for every $s \in \mathbb{N}$. Furthermiore, $U_{z}$ satisfies the boundary conditions (3.24) for $0 \leqslant s \leqslant m-1$. This implies $U_{z} \in h_{m}(\Omega)$, and hence $U_{z} \in D(A)$. Since $U_{z}$ satisfies (3.1), we obtain $U_{2}=R_{z} f$. We collect these results in the following lemma:

Lemma 3.1. Assume that $f \in C_{0}^{\infty}(\Omega)$ and $z \in \mathbb{C} \backslash\left[\lambda_{1}, \infty\right)$. Then the Fourier coefficients $u_{j k}$ of $R_{z} f$ are given by (3.11), (3.5) and (3.10), and the Fourier series (3.2) of $R_{z} f$ and its termwise derivatives of arbitrary order converge uniformly with respect to $\mathrm{x} \in \overline{\mathbf{\Omega}}$ and $z \in K$ for every compact subset $K$ of $\mathbb{C} \backslash\left[\lambda_{1}, \infty\right)$.

\section{The spectral family}

In order to compute the spectral family $\left\{P_{\lambda}\right\}$ of $A$, we investigate the behaviour of $R_{z} f$ as $\operatorname{Im} z \rightarrow 0$. Consider the Fourier series (3.2) of $R_{z} f$ and set $z=\rho+i \tau$ with $\rho, t \in \mathbb{R}$. According to (3.12), the Fourier coefficients $u_{j k}$ of $R_{\rho+i \tau} f$ are solutions of

$$
\left[L(m)+\lambda_{j}-\rho-\mathrm{i} \tau\right] u_{j k}(. ; \rho+\mathrm{i} \tau)=f_{j k} .
$$

Initially we assume $\lambda_{j}>\rho$. In this case (4.1) has a uniquely determined solution also for $\tau=0$, since $L(m)$ is positive and self-adjoint. This solution $u_{j k}(. ; \rho)$ is given by (3.10) and (3.11). We set

$$
S(x, \rho+\mathrm{i} \tau):=\sum_{j=\imath(\rho)+1}^{\infty} \sum_{k=1}^{\kappa(j)} u_{j k}(x ; \rho+\mathrm{i} \tau) V_{j k}(y),
$$

where

$$
l(\rho):= \begin{cases}\max \left\{s \in \mathbb{N}: \lambda_{s} \leqslant \rho\right\} & \text { if } \rho \geqslant \lambda_{1}, \\ 0 & \text { if } \rho<\lambda_{1}\end{cases}
$$


From (3.15), (3.18) and (3.22) it follows that the series (4.2) converges uniformly with respect to $x \in \bar{\Omega}$ and $\tau \in \mathbb{R}$. It is our first aim to estimate the difference $S(x ; \rho+i \tau)$ $-S(\mathbf{x} ; \rho)$. We conclude from (4.1) that

$$
\left[L(m)+\lambda_{r}-\rho\right]\left[u_{j k}(. ; \rho+\mathrm{i} \tau)-u_{j k}(; \rho)\right]=\mathrm{i} \tau u_{j k}(. ; \rho+\mathrm{i} \tau) .
$$

The argument leading to (3.17) yields

$$
\left\|u_{j k}(. ; \rho+\mathrm{i} \tau)-u_{j k}(. ; \rho)\right\|_{s, \mathbb{R}^{\prime}} \leqslant \frac{\tau}{\lambda_{j}-\rho}\left\|u_{j k}(. ; \rho+\mathrm{i} \tau)\right\|_{s, \mathbb{R}^{\prime}}
$$

for every $s \in \mathbb{N}$. Combining this with (3.17), we have

$$
\left\|u_{j k}(. ; \rho+i \tau)-u_{j k}(. ; \rho)\right\|_{s, \mathbb{R}^{i}} \leqslant \frac{\tau}{\left(\lambda_{j}-\rho\right)^{2}}\left\|f_{j k}\right\|_{s, \mathbb{R}^{\prime}} .
$$

A consideration analogous to that before (3.22) shows that

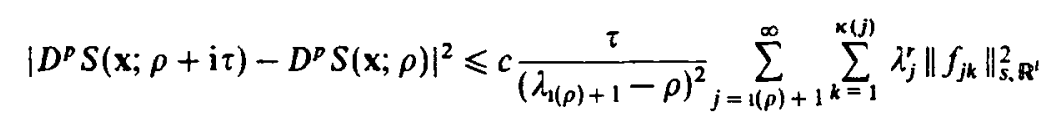

with suitable constants $c>0$ and $r, s \in \mathbb{N}$ (depending on $p \in \mathbb{N}_{0}^{n}$ ). This implies

$$
D^{p} S(\mathbf{x} ; \rho+\mathrm{i} \tau)-D^{p} S(\mathbf{x} ; \rho)=O(\tau) \quad \text { as } \tau \rightarrow 0
$$

for every $p \in \mathbb{N}_{0}^{n}$ uniformly with respect to $x \in \bar{\Omega}$.

The discussion of the Fourier coefficients $u_{j k}$ with $j \leqslant i(\rho)$ is based on their representation (3.11). First we study the case $\lambda_{j}<\rho$. According to the definition (3.10) of $\varphi_{s}$ we have

$$
\left.\begin{array}{l}
\varphi_{s}(\rho+\mathrm{i} 0 ; j):=\lim _{\tau \downarrow 0} \varphi_{s}(\rho+\mathrm{i} \tau ; j)=\frac{2 \pi s}{m}, \\
\varphi_{s}(\rho-\mathrm{i} 0 ; j):=\lim _{\tau \uparrow 0} \varphi_{s}(\rho+\mathrm{i} \tau ; j)=\frac{2 \pi(s+1)}{m} .
\end{array}\right\}
$$

From this and (3.11) it follows that $u_{j k}(x ; \rho+\mathrm{i} \tau)$ converges uniformly with respect to $x$ in every compact subset $M$ of $\mathbb{R}^{l}$ as $\tau \downarrow 0$ and $\tau \uparrow 0$, respectively. The limit functions $u_{j k}(x ; \rho+\mathrm{i} 0)$ and $u_{j k}(x ; \rho-\mathrm{i} 0)$ are given by (3.11) with $\varphi_{s}$ defined by (4.5). In the same way we conclude from (3.16) that $D^{p} u_{j k}(x ; \rho+\mathrm{i} \tau)$ with arbitrary $p \in \mathbb{N}_{0}^{l}$ converges uniformly in $M$ to $D^{p} u_{j k}(x ; \rho+\mathrm{i} 0)$ and $D^{p} u_{j k}(x ; \rho-\mathrm{i} 0)$ as $\tau \downarrow 0$ and $\tau \uparrow 0$, respectively. Thus we obtain by (4.1)

$$
\left[\left(-\Delta_{x}\right)^{m}+\lambda_{j}-\rho\right] u_{j k}(x ; \rho \pm \mathrm{i} 0)=f_{j k}(x) \text { for } x \in \mathbb{R}^{l} \text { and } \lambda_{j}<\rho .
$$

Now we study the case $\lambda_{j}=\rho$. In this case we have

$$
\varphi_{s}=\frac{\arg (\mathrm{i} \tau)+2 \pi s}{m}=\left(2 s+1-\frac{\operatorname{sign} \tau}{2}\right) \frac{\pi}{m} .
$$

We use the series representations

$$
J_{\sigma}(\zeta)=\zeta^{\sigma} \sum_{s=0}^{\infty} C_{s} \zeta^{2 s}
$$




$$
N_{\sigma}(\zeta)= \begin{cases}\zeta^{-\sigma} \sum_{s=0}^{\infty} C_{s}^{\prime} \zeta^{2 s} & \left(\sigma+\frac{1}{2} \in \mathbb{N}_{0}\right), \\ \frac{2}{\pi} J_{\sigma}(\zeta) \ln \frac{\gamma \zeta}{2}+\zeta^{\sigma} \sum_{s=0}^{\infty} C_{s}^{\prime \prime} \zeta^{2 s}+\zeta^{-\sigma} \sum_{s=0}^{\circ-1} C_{s}^{\prime \prime \prime} \zeta^{2 s} & \left(\sigma \in \mathbb{N}_{0}\right)\end{cases}
$$

for $|\arg \zeta|<\pi$, where $\ln \gamma=C_{\mathrm{e}}$ (the Euler-Mascheroni constant) and

$$
\begin{aligned}
& C_{s}:=\frac{(-1)^{s}}{2^{\sigma+2 s} s ! \Gamma(\sigma+s+1)}, \\
& C_{s}^{\prime}:=\frac{(-1)^{\sigma+s+\frac{1}{2}}}{2^{2 s-\sigma} s ! \Gamma(s+1-\sigma)}, \\
& C_{s}^{\prime \prime}:=\frac{(-1)^{s+1}}{\pi 2^{\sigma+2 s} s !(\sigma+s) !}\left(\sum_{r=1}^{s} \frac{1}{r}+\sum_{r=1}^{s+\sigma} \frac{1}{r}\right), \\
& C_{s}^{\prime \prime}:=-\frac{2^{\sigma-s}(\sigma-s-1) !}{\pi s !}
\end{aligned}
$$

(compare Reference 5). We insert these expansions into (3.11) and change the order of integration and summation. This leads to terms of the form

$$
w_{j k}[h(\zeta)]:=\frac{\mathrm{i}|\tau|^{\mid+2}-1}{4 m(2 \pi)^{\sigma}} \sum_{r=0}^{m-1} \mathrm{e}^{\mathrm{i}[(\sigma / 2)+1-m] \varphi_{r}} \int_{R^{\prime}} \frac{f_{j k}\left(x^{\prime}\right)}{\left|x-x^{\prime}\right|^{\sigma}} h\left(\left|x-x^{\prime}\right||\tau|^{1 / 2 m} \mathrm{e}^{\mathrm{j} \varphi_{r} / 2}\right) \mathrm{d} x^{\prime},
$$

where $h(\zeta)=\zeta^{2 s+\sigma}, h(\zeta)=\zeta^{2 s-\sigma}$ and $h(\zeta)=\zeta^{2 s+\sigma} \ln \zeta$, respectively. Computing the terms $w_{j k}\left[\zeta^{2 s+\sigma}\right]$, we obtain

$$
\begin{aligned}
& w_{j k}\left[\zeta^{2 s+\sigma}\right]=\frac{\mathrm{i}|\tau|^{\frac{\alpha+1+3}{m}-1}}{4 m(2 \pi)^{\sigma}} \int_{\mathbb{R}^{\prime}} f_{j k}\left(x^{\prime}\right)\left|x-x^{\prime}\right|^{2 s} \mathrm{~d} x^{\prime} \sum_{r=0}^{m-1} \mathrm{e}^{\mathrm{i}(\sigma+1+s-m) \varphi,} \\
& = \begin{cases}0 & \text { for } \sigma \in \mathbb{N}_{0} ; s=0, \ldots, m-\sigma-2, \\
\frac{\mathrm{i}}{4(2 \pi)^{\sigma}} \int_{\mathbb{R}^{\prime}} f_{j k}\left(x^{\prime}\right)\left|x-x^{\prime}\right|^{2(m-\sigma-1)} \mathrm{d} x^{\prime} & \text { for } \sigma \in \mathbb{N}_{0} ; s=m-\sigma-1, \\
\frac{D_{s}}{|\tau|^{1-(\sigma+s+1) / m}} \int_{\mathbb{R}^{\prime}} f_{j k}\left(x^{\prime}\right)\left|x-x^{\prime}\right|^{2 s} \mathrm{~d} x^{\prime} & \text { for } \sigma+\frac{1}{2} \in \mathbb{N}_{0} ; \quad s=0, \ldots, m-\sigma-\frac{3}{2},\end{cases}
\end{aligned}
$$

with

$$
D_{s}:=\frac{\mathrm{i}}{2 m(2 \pi)^{\sigma}} \frac{\mathrm{e}^{\mathrm{i} \pi(\sigma+s+1-m)(2-\operatorname{sign} \pi) / 2 m}}{1-\mathrm{e}^{\mathrm{i} 2 \pi(\sigma+1+s-m) / m}} .
$$

It follows in the same way that

$$
w_{j k}\left[\zeta^{2 s-\sigma}\right]= \begin{cases}0 & \text { if } s=0, \ldots, m-2, \\ \frac{\mathrm{i}}{4(2 \pi)^{\sigma}} \int_{\mathbb{R}^{\prime}} f_{j k}\left(x^{\prime}\right)\left|x-x^{\prime}\right|^{2(m-\sigma-1)} \mathrm{d} x^{\prime} & \text { if } s=m-1 .\end{cases}
$$


708

P. Leaky Jr.

Since

$$
\sum_{r=0}^{m-1} \varphi_{r} \mathrm{e}^{\mathrm{i} v \varphi_{r}}= \begin{cases}\pi\left(m-\frac{\operatorname{sign} \tau}{2}\right) & \text { if } v=0, \\ -\frac{2 \pi \mathrm{e}^{\mathrm{i} v \pi(2-\operatorname{sign\tau } \tau) / 2 m}}{1-\mathrm{e}^{\mathrm{i} 2 v \pi / m}} & \text { if } v=-1, \ldots,-(m-1),\end{cases}
$$

we obtain for $\sigma \in \mathbb{N}_{0}$

$$
\begin{aligned}
& \frac{2}{\pi} w_{j k}\left[\zeta^{2 s+\sigma} \ln \zeta\right]=\frac{\mathrm{i}|\tau|^{\alpha+1+1}-1}{m(2 \pi)^{\sigma+1}}\left[\int_{\mathbf{R}^{\prime}} f_{j k}\left(x^{\prime}\right)\left|x-x^{\prime}\right|^{2 s} \mathrm{~d} x^{\prime} \sum_{r=0}^{m-1} \mathrm{i}^{\varphi_{r}} \mathrm{e}^{\mathrm{i}(\sigma+s+1-m) \varphi_{r}}\right. \\
& \left.\quad+\int_{\mathbf{R}^{\prime}} f_{j k}\left(x^{\prime}\right)\left|x-x^{\prime}\right|^{2 s} \ln \left(\left|x-x^{\prime}\right||\tau|^{1 / 2 m}\right) \mathrm{d} x^{\prime} \sum_{r=0}^{m-1} \mathrm{e}^{\mathrm{i}(\sigma+s+1-m) \varphi_{r}}\right] \\
& = \begin{cases}-\frac{\mathrm{i} D_{s}}{|\tau|^{1-\frac{\sigma+s+1}{m}}} \int_{\mathbf{R}^{\prime}} f_{j k}\left(x^{\prime}\right)\left|x-x^{\prime}\right|^{2 s} \mathrm{~d} x^{\prime} & \text { if } s=0, \ldots, m-\sigma-2, \\
\left(\frac{\mathrm{i} \ln |\tau|}{2 m(2 \pi)^{\sigma+1}}-\frac{2 m-\operatorname{sign} \tau}{8 m(2 \pi)^{\sigma}}\right) \int_{\mathbf{R}^{\prime}} f_{j k}\left(x^{\prime}\right)\left|x-x^{\prime}\right|^{2(m-\sigma-1)} \mathrm{d} x^{\prime} & \\
+\frac{\mathrm{i}}{(2 \pi)^{\sigma+1}} \int_{\mathbf{R}^{\prime}} f_{j k}\left(x^{\prime}\right)\left|x-x^{\prime}\right|^{2(m-\sigma-1)} \ln \left|x-x^{\prime}\right| \mathrm{d} x^{\prime} & \text { if } s=m-\sigma-1 .\end{cases}
\end{aligned}
$$

Note that $\sigma=l / 2-1$. Inserting (4.8)-(4.14) in (3.11) we obtain for odd $l$

$$
\begin{aligned}
u_{j k}\left(x ; \lambda_{j}+i \tau\right)= & \sum_{s=0}^{m-(l+1) / 2} \frac{C_{s} D_{s}}{|\tau|^{1-(l+2 s) / 2 m}} \int_{\mathbf{R}^{\prime}} f_{j k}\left(x^{\prime}\right)\left|x-x^{\prime}\right|^{2 s} \mathrm{~d} x^{\prime} \\
& -\frac{C_{m-1}^{\prime}}{4(2 \pi)^{(l / 2)-1}} \int_{\mathbf{R}^{\prime}} f_{j k}\left(\mathrm{x}^{\prime}\right)\left|x-x^{\prime}\right|^{2 m-1} \mathrm{~d} x^{\prime} \\
& +O\left(|\tau|^{1 / 2 m}\right) \text { as }|\tau| \rightarrow 0
\end{aligned}
$$

and for even $l$

$$
\begin{aligned}
u_{j k}\left(x ; \lambda_{j}+\mathrm{i} \tau\right)= & \sum_{s=0}^{m-1-(1 / 2)} \frac{C_{s} D_{s}}{|\tau|^{1-(l+2 s) / 2 m}} \int_{\mathbb{R}^{\prime}} f_{j k}\left(x^{\prime}\right)\left|x-x^{\prime}\right|^{2 s} \mathrm{~d} x^{\prime} \\
& -\left(\frac{C_{m-(l / 2)}}{2 m(2 \pi)^{l / 2}} \ln |\tau|+D^{\prime}(\operatorname{sign} \tau)\right) \int_{\mathbf{R}^{\prime}} f_{j k}\left(x^{\prime}\right)\left|x-x^{\prime}\right|^{2 m-1} \mathrm{~d} x^{\prime} \\
& -\frac{C_{m-(l / 2)}}{(2 \pi)^{1 / 2}} \int_{R^{\prime}} f_{j k}\left(x^{\prime}\right)\left|x-x^{\prime}\right|^{2 m-l} \ln \left|x-x^{\prime}\right| \mathrm{d} x^{\prime} \\
& +O\left(|\tau|^{1 / 2 m}\right) \text { as }|\tau| \rightarrow 0,
\end{aligned}
$$

with

$$
D^{\prime}(\operatorname{sign} \tau):=\frac{1}{4(2 \pi)^{(1 / 2)-1}}\left(C_{m-(l / 2)}\left(\frac{2}{\pi} \ln \frac{\gamma}{2}-\mathrm{i} \frac{\operatorname{sign} \tau}{2 m}\right)+C_{m-(l / 2)}^{\prime \prime}+C_{m-1}^{\prime \prime \prime}\right)
$$


$\left(C_{s}=C_{s}^{\prime}=C_{s}^{\prime \prime}=C_{s}^{\prime \prime \prime}:=0\right.$ if $s<0, C_{s}^{\prime \prime \prime}:=0$ if $\left.s>l / 2\right)$. Both asymptotic formulae (4.15) and (4.16) hold uniformly in every compact subset of $\mathbb{R}^{l}$. Hence, $u_{j k}$ may be unbounded as $|\tau| \rightarrow 0$. But if $l>2 m$, then (4.15) and (4.16) imply

$$
u_{j k}\left(x ; \lambda_{j}+\mathrm{i} \tau\right) \rightarrow u_{j k}\left(x ; \lambda_{j}\right):=\frac{\Gamma(l / 2-m)}{\pi^{l / 2} 4^{m}(m-1) !} \int_{R^{\prime}} \frac{f_{j k}\left(x^{\prime}\right)}{\left|x-x^{\prime}\right|^{j-2 m}} \mathrm{~d} x^{\prime}
$$

as $\tau \rightarrow 0$. If $l \leqslant 2 m$ and

$$
\int_{\mathbb{R}^{\prime}} f_{j k}\left(x^{\prime}\right)\left|x-x^{\prime}\right|^{2 s} \mathrm{~d} x^{\prime}=0 \text { for } x \in \mathbb{R}^{l} ; s=0, \ldots,[m-l / 2]
$$

$([\alpha]:=\max \{i \in \mathbb{N}: i \leqslant \alpha\})$, then the limit $u_{j k}\left(x ; \lambda_{j}\right)$ exists also according to $(4.15)$ and (4.16), and we have

$$
u_{j k}\left(x ; \lambda_{j}\right):= \begin{cases}\frac{\Gamma(l / 2-m)}{\pi^{l / 2} 4^{m}(m-1) !} \int_{R^{\prime}} f_{j k}\left(x^{\prime}\right)\left|x-x^{\prime}\right|^{2 m-l} \mathrm{~d} x^{\prime} & \text { if } l \text { is odd } \\ \frac{(-1)^{m+1-(1 / 2)}}{\pi^{l / 2} 2^{2 m-1}(m-l / 2) !(m-1) !} \int_{\mathbf{R}^{\prime}} f_{j k}\left(x^{\prime}\right)\left|x-x^{\prime}\right|^{2 m-l} \ln \left|x-x^{\prime}\right| \mathrm{d} x^{\prime} & \text { if } l \text { is even. }\end{cases}
$$

Now we consider the condition (4.19). It is equivalent to

$$
0=\sum_{\alpha+\beta+\gamma=s} \frac{s !}{\alpha ! \beta ! \gamma !}|x|^{2 \alpha} \int_{\mathbb{R}^{\prime}} f_{j k}\left(x^{\prime}\right)\left(-2 x \cdot x^{\prime}\right)^{\beta}\left|x^{\prime}\right|^{2 y} \mathrm{~d} x^{\prime}
$$

for $x \in \mathbb{R}^{l}, s=0, \ldots,[m-l / 2]$. By induction with respect to $s$ we obtain

$$
\sum_{\beta+\gamma=s} \frac{(-2)^{\beta} s !}{\beta ! \gamma !} \int_{R^{\prime}} f_{j k}\left(x^{\prime}\right)\left(x \cdot x^{\prime}\right)^{\beta}\left|x^{\prime}\right|^{2 \gamma} \mathrm{d} x^{\prime}=0
$$

for $x \in \mathbb{R}^{l}, s=0, \ldots,[m-l / 2]$. Note that for $p \in \mathbb{N}_{0}^{l}$

$$
\begin{aligned}
& {\left[D_{x}^{p} \int_{\mathbf{R}^{\prime}} f_{j k}\left(x^{\prime}\right)\left(x \cdot x^{\prime}\right)^{\beta}\left|x^{\prime}\right|^{2 y} \mathrm{~d} x^{\prime}\right]_{x=0}} \\
& = \begin{cases}0 & \text { if }|p| \neq \beta, \\
\beta ! \int_{\mathbf{R}^{\prime}} f_{j k}\left(x^{\prime}\right) x^{\prime p}\left|x^{\prime}\right|^{2 \gamma} \mathrm{d} x^{\prime} & \text { if }|p|=\beta,\end{cases}
\end{aligned}
$$

since $D_{x}^{p}\left(x \cdot x^{\prime}\right)^{\beta}=\beta \cdot \ldots \cdot(\beta-|p|+1)\left(x \cdot x^{\prime}\right)^{\beta-|p|} x^{\prime p}$. Therefore (4.19) implies

$$
\int_{\mathbb{R}^{\prime}} f^{j k}\left(x^{\prime}\right) x^{\prime p}\left|x^{\prime}\right|^{2 s} \mathrm{~d} x^{\prime}=0 \text { for } p \in \mathbb{N}_{0}^{l}, s \in \mathbb{N}_{0} \text { with }|p|+s \leqslant[m-l / 2] .
$$

By (4.21) also the inverse implication holds, so that (4.19) is equivalent to (4.22).

We summarize our results: the limit of $R_{\rho+i \tau} f$ as $\tau \downarrow 0$ exists if and only if one of the conditions

$$
\begin{aligned}
& l>2 m, \\
& l \leqslant 2 m \text { and } \rho \neq \lambda_{j} \text { for every } j \in \mathbb{N},
\end{aligned}
$$


$l \leqslant 2 m, \rho=\lambda_{j}$ and (4.22) holds for $k=1,2, \ldots, \kappa(j)$

is valid. In this case $R_{\rho+\mathrm{i}} f$ converges also as $\tau \uparrow 0$. The limits have the Fourier series expansions

$$
R_{\rho \pm i 0} f(\mathbf{x})=\sum_{j=1}^{\prime(\rho)} \sum_{k=1}^{\kappa(j)} u_{j k}(x ; \rho \pm \mathrm{i} 0) V_{j k}(y)+\sum_{j=t(\rho)+1}^{\infty} \sum_{k=1}^{\kappa(j)} u_{j k}(x ; \rho) V_{j k}(y)
$$

where $u_{j k}$ is given by (3.11) and (3.10) if $\lambda_{j}>\rho,(3.11)$ and (4.5) if $\lambda_{j}<\rho$ and (4.18) or (4.20) if $\lambda_{j}=\rho$, respectively. The series (4.26) converges uniformly with respect to $\mathbf{x}=(x, y)$ in every compact subset $M$ of $\bar{\Omega}$. In the same way we obtain $D^{p} R_{\rho+\mathrm{i} \tau} f(\mathbf{x}) \rightarrow D^{p} R_{\rho+\mathrm{i} 0} f(\mathbf{x})$ as $\tau \downarrow 0$ and $D^{p} R_{\rho+\mathrm{i} z} f(\mathbf{x}) \rightarrow D^{p} R_{\rho-\mathrm{i} 0} f(\mathbf{x})$ as $\tau \uparrow 0$ uniformly with respect to $\mathrm{x} \in M\left(p \in \mathbb{N}_{0}^{n}\right)$. Together with (3.1) this yields that $R_{p+\mathrm{i} 0} f$ and $R_{\rho-\mathrm{i} 0} f$ are solutions of the boundary value problem

$$
\begin{array}{ll}
{\left[\left(-\Delta_{x}\right)^{m}+\left(-\Delta_{y}\right)^{m}-\rho\right] w=f} & \text { in } \Omega, \\
w=\frac{\partial w}{\partial \mathbf{n}}=\ldots=\frac{\partial^{m-1} w}{\partial \mathbf{n}^{m-1}}=0 & \text { on } \partial \Omega .
\end{array}
$$

We conclude these considerations by deriving a condition that characterizes $R_{\rho+\text { io }} f$ uniquely among the solutions of (4.27). Let $w \in C^{2 m}(\bar{\Omega})$ be an arbitrary solution of (4.27) and set $w_{j k}(x):=\int_{\Omega^{\prime}} w(x, y) V_{j k}(y) \mathrm{d} y$. The boundary condition in (4.27) implies that $w(x,.) \in \stackrel{\circ}{m}_{m}\left(\Omega^{\prime}\right) \cap H_{2 m}\left(\Omega^{\prime}\right)$ for every $x \in \mathbb{R}^{l}$. The expansion theorem for the interior boundary value problem (1.11) yields

$$
w(x, .)=\sum_{j=1}^{\infty} \sum_{k=1}^{x(j)} w_{j k}(x) V_{j k},\left(-\Delta_{y}\right)^{m} w(x, .)=\sum_{j=1}^{\infty} \sum_{k=1}^{\kappa(j)} \lambda_{j} w_{j k}(x) V_{j k} .
$$

On the other hand, we have

$$
\left(-\Delta_{x}\right)^{m} w(x, .)=\sum_{j=1}^{\infty} \sum_{k=1}^{x(j)} w_{j k}^{*}(x) V_{j k}
$$

with $w_{j k}^{*}(x)=\int_{\Omega^{\prime}}\left(-\Delta_{x}\right)^{m} w(x, y) V_{j k}(y) \mathrm{d} y=\left(-\Delta_{x}\right)^{m} w_{j k}(x)$. Inserting this and the Fourier expansion (3.4) of $f$ into (4.27), we obtain

$$
\left[\left(-\Delta_{x}\right)^{m}+i_{j}-\rho\right] w_{j k}=f_{j k} \quad \text { in } \mathbb{R}^{l}
$$

for $j=1,2, \ldots$ and $k=1,2, \ldots \kappa(j)$. The Fourier coefficients $u_{j k}$ of $R_{\rho+i 0} f$ can be uniquely characterized in the following way: if $\lambda_{j}>\rho$, then $u_{j k}$ is uniquely determined by (4.28) and the property $u_{j k} \in D(L(m))$, since $L(m)$ is self-adjoint and positive. If $\lambda_{j}<\rho$, it can be shown that $u_{j k}$ is the only solution of (4.28) satisfying

$$
\left.\begin{array}{ll}
\Delta_{x}^{s} u_{j k}(x ; \rho+\mathrm{i} 0)=o\left(\frac{1}{r^{(1-1) / 2}}\right) & \text { as } r=|x| \rightarrow \infty, \\
\left(\frac{\partial}{\partial r}-\mathrm{i}\left|\lambda_{j}-\rho\right|^{1 / 2 m}\right) \Delta_{x}^{s} u_{j k}(x ; \rho+\mathrm{i} 0)=o\left(\frac{1}{r^{(1-1) / 2}}\right) & \text { as } r=|x| \rightarrow \infty
\end{array}\right\}
$$

for $s=0, \ldots, m-1$. Formula (4.29) can be considered as a generalization of Sommerfeld's radiation condition. If $\lambda_{j}=\rho$ and (4.22) holds, then $u_{j k}$ may be unbounded as $|x| \rightarrow \infty$. It will be shown in Reference 4 that $u_{j k}$ is the only solution of 
(4.28) with

$$
\int_{\left|x-x_{0}\right|=R} u_{j k}(x ; \rho) \mathrm{d} S_{x}=o\left(R^{l-1}\right) \quad \text { as } R \rightarrow \infty \text { for every } x_{0} \in \mathbb{R}^{l} .
$$

Hence, $R_{\rho+i_{0}} f$ is uniquely determined by (4.27) and the properties of its Fourier coefficients $u_{j k}$ collected above. Thus we have proved:

Lemma 4.1. Assume that $f \in C_{0}^{\infty}(\Omega)$ and $\rho \in \mathbb{R}$. The resolvent $R_{\rho+\mathrm{i}} f$ of the operator $A$ converges as $\tau \downarrow 0$ if and only if one of the conditions (4.23)-(4.25) holds. The limit function $R_{\rho+i 0} f$ is the uniquely determined solution of (4.27) with the property that the Fourier coefficients $u_{j k}$ belong to $D(L(m))$ if $\lambda_{j}>\rho$ and satisfy the condition (4.29) for $s=0, \ldots, m-1$ if $\lambda_{j}<\rho$ or $(4.30)$ if $\lambda_{j}=\rho$, respectively.

Our next aim is to construct of the spectral family $\left\{P_{\lambda}\right\}$ of $A$. First we summarize some properties of $R_{z} f$ obtained above:

(i) We have

$$
R_{\rho+\mathrm{i} \tau} f(\mathrm{x})-R_{\rho-\mathrm{i} \tau} f(\mathrm{x}) \rightarrow R_{\rho+\mathrm{i} 0} f(\mathrm{x})-\boldsymbol{R}_{\rho-\mathrm{i} 0} f(\mathrm{x}) \quad \text { as } \tau \downarrow 0
$$

uniformly with respect to $(\mathbf{x}, \rho) \in M \times K$, where $M$ and $K$ are arbitrary compact subsets of $\bar{\Omega}$ and $\mathbb{R} \backslash\left\{\lambda_{j}: j \in \mathbb{N}\right\}$, respectively.

(ii) From (3.11), (3.9), (4.8) and (4.9) we obtain

$$
u_{j k}(x ; z)=o\left(\frac{\ln \left|z-\lambda_{j}\right|}{\left|z-\lambda_{j}\right|^{1-(1 / 2 m)}}\right) \quad \text { as } z \rightarrow \lambda_{j}
$$

and by (4.2) and (4.4)

$$
R_{z} f(\mathbf{x})-R_{\bar{z}} f(\mathbf{x})=O\left(\frac{\ln \left|z-\hat{\lambda}_{j}\right|}{\left|z-\lambda_{j}\right|^{1-(1 / 2 m)}}\right) \quad \text { as } z \rightarrow \lambda_{j}
$$

uniformly in every compact subset $M$ of $\bar{\Omega}$.

We conclude from (i) and (ii) by the same argument used in the verification of formula (2.36) in Reference 12 that

$$
\left(P_{\lambda} f\right)(\mathbf{x})=\frac{1}{2 \pi \mathrm{i}} \int_{0}^{\lambda}\left[R_{\rho+\mathrm{i} 0} f(\mathbf{x})-R_{p-\mathrm{i} 0} f(\mathbf{x})\right] \mathrm{d} \rho
$$

and that $P_{\lambda}$ is continuous with respect to $\lambda$. In particular, $A$ has no eigenvalues.

In order to compute the integrand of (4.33), its convenient to set

$$
\left.\begin{array}{rl}
u^{(j)}(\mathbf{x} ; z) & :=\sum_{k=1}^{\kappa(j)} u_{j k}(x) V_{j k}(y), \\
f^{(j)}(\mathbf{x} ; z) & :=\sum_{k=1}^{\kappa(j)} f_{j k}(x) V_{j k}(y) .
\end{array}\right\}
$$

Relation (4.26) implies

$$
R_{\rho+\mathrm{j} 0} f(\mathbf{x})-R_{\rho-\mathrm{i} 0} f(\mathbf{x})=\sum_{j=1}^{i(p)}\left[u^{(j)}(\mathrm{x} ; \rho+\mathrm{i} 0)-u^{(j)}(\mathbf{x} ; \rho-\mathrm{i} 0)\right]
$$

Since

$$
\begin{aligned}
& \varphi_{r+1}(\rho+\mathrm{i} 0 ; j)=\varphi_{r}(\rho-\mathrm{i} 0 ; j) \quad \text { for } r=0, \ldots, m-2 \\
& \varphi_{0}(\rho+\mathrm{i} 0 ; j)=0, \quad \varphi_{m-1}(\rho-\mathrm{i} 0 ; j)=2 \pi
\end{aligned}
$$


(compare (4.5)), it follows from the representation (3.11) of $u_{j k}$ that

$$
\begin{aligned}
& u^{(j)}(\mathrm{x} ; \rho+\mathrm{i} 0)-u^{(j)}(\mathrm{x} ; \rho-\mathrm{i} 0) \\
& =\frac{\mathrm{i}\left|\rho-\lambda_{j}\right|^{\rho+2}-1}{4 m(2 \pi)^{\sigma}} \int_{\mathbf{R}^{\prime}} \frac{f^{(j)}\left(x^{\prime}, y\right)}{\left|x-x^{j}\right|^{\sigma}}\left\{H _ { \sigma } ^ { ( 1 ) } \left(\left|x-x^{\prime}\right|\left|\rho-\lambda_{j}\right|^{1 / 2 m}\right.\right. \\
& \left.-\mathrm{e}^{\mathrm{i} \sigma x} H_{\sigma}^{(1)}\left(-\left|x-x^{\prime}\right|\left|\rho-\lambda_{j}\right|^{3 / 2 m}\right)\right\} \mathrm{d} x^{\prime}
\end{aligned}
$$

if $\lambda_{j}<\rho$. Using

$$
J_{\sigma}(-\zeta)=\mathrm{e}^{\mathrm{i} \sigma \pi} J_{\sigma}(\zeta), \quad N_{\sigma}(-\zeta)=\mathrm{e}^{-\mathrm{i} \sigma \pi} N_{\sigma}(\zeta)+2 \mathrm{i} \cos (\sigma \pi) J_{\sigma}(\zeta)
$$

(compare Reference 5), we obtain

$$
\begin{aligned}
u^{(j)}(\mathrm{x} ; \rho+\mathrm{i} 0)-u^{(j)}(\mathbf{x} ; \rho-\mathrm{i} 0)= & \frac{\mathrm{i}\left|\rho-\lambda_{j}\right|^{\frac{\rho^{2} 2}{2 m}}-1}{2 m(2 \pi)^{\sigma}} \int_{R^{\prime}} \frac{f^{(j)}\left(x^{\prime}, y\right)}{\left|x-x^{\prime}\right|^{\sigma}} \\
& \times J_{\sigma}\left(\left|x-x^{\prime}\right|\left|\rho-\lambda_{j}\right|^{1 / 2 m}\right) \mathrm{d} x^{\prime}
\end{aligned}
$$

for $\lambda_{j}<\rho$. Thus the spectral family of $A$ is given by the improper integral

$$
P_{\lambda} f(\mathbf{x})=\int_{0}^{\lambda}\left\{\sum_{j=1}^{\prime(\rho)} \frac{\left|\rho-\lambda_{j}\right|^{\mid+2}-1}{2 m(2 \pi)^{\sigma+1}} \int_{\mathbf{R}^{k}} \frac{f^{(j)}\left(x^{\prime}, y\right)}{\left|x-x^{\prime}\right|^{\sigma}} J_{\sigma}\left(\left|x-x^{\prime}\right|\left|\rho-\lambda_{j}\right|^{1 / 2 m}\right) \mathrm{d} x^{\prime}\right\} \mathrm{d} \rho .
$$

This formula shows that $P_{\lambda}=0$ for $\lambda \leqslant \lambda_{1}$. Furthermore, it can be shown in the same way as in Reference 12, page 186, that the spectrum $\sigma(A)$ of $A$ consists of the interval $\left[\lambda_{1}, \infty\right)$. If $\lambda \neq \lambda_{1}, \lambda_{2}, \ldots$, then we have by (4.37)

$$
\frac{\mathrm{d} P_{\lambda} f(\mathbf{x})}{\mathrm{d} \lambda}=\frac{1}{2 m(2 \pi)^{\sigma+1}} \sum_{j=1}^{(1 \lambda)}\left|\lambda-\lambda_{j}\right|^{\frac{\beta}{2 m}+2}-1 \int_{\mathbf{R}^{\prime}} \frac{f^{(j)}\left(x^{\prime}, y\right)}{\left|x-x^{\prime}\right|^{\sigma}} J_{\sigma}\left(\left|x-x^{\prime}\right|\left|\lambda-\lambda_{j}\right|^{1 / 2 m}\right) \mathrm{d} x^{\prime} .
$$

If $l \leqslant 2 m$ or, equivalently, $\sigma+1 \leqslant m$, then $P_{\lambda} f$ is not differentiable at $\lambda=\lambda_{k}(k \in \mathbb{N})$. Inserting (4.8) into (4.38), we obtain

$$
\frac{\mathrm{d} P_{\lambda} f(\mathbf{x})}{\mathrm{d} \lambda}= \begin{cases}g_{k}(\mathbf{x})+O\left(\left|\lambda-\lambda_{k}\right|\right) & \text { as } \lambda \uparrow \lambda_{k}, \\ \frac{1}{2 m(2 \pi)^{\sigma+1}} \sum_{s=0}^{(m-\sigma-1)} \frac{C_{s}}{\left|\lambda-\lambda_{k}\right|^{1-(\sigma+1+s) / m}} \int_{\mathbb{R}^{\prime}} f^{(k)}\left(x^{\prime}, y\right)\left|x-x^{\prime}\right|^{2 s} \mathrm{~d} x^{\prime} & \\ +g_{k}(\mathbf{x})+O\left(\left|\lambda-\lambda_{k}\right|^{1 / 2 m}\right) & \text { as } \lambda \downarrow \lambda_{k}\end{cases}
$$

uniformly in every compact subset $M$ of $\bar{\Omega}$, where

$$
g_{k}(\mathbf{x})=\frac{1}{2 m(2 \pi)^{\sigma+1}} \sum_{j=1}^{k-1} \mid \lambda_{k}-\lambda_{j}^{\mid \gamma^{+\frac{2}{2 m}}-1} \int_{\mathbf{R}^{\prime}} \frac{f^{(j)}\left(x^{\prime}, y\right)}{\left|x-x^{\prime}\right|^{\sigma}} J_{\sigma}\left(\left|x-x^{\prime}\right|\left|\lambda_{k}-\lambda_{j}\right|^{1 / 2 m}\right) \mathrm{d} x^{\prime}
$$

If $l>2 m$, then $P_{\lambda} f$ is continuously differentiable with respect to $\lambda \in \mathbb{R}$. We collect our results in the following lemma:

\section{Lemma 4.2.}

(i) The spectral family $P_{\lambda}$ of $A$ is continuous on $\mathbb{R}$ and vanishes for $\lambda \leqslant \lambda_{1} ; P_{\lambda} f$ is given by (4.37) if $f \in C_{0}^{\infty}(\Omega)$. 
(ii) The operator $A$ has no eigenvalues. The spectrum $\sigma(A)$ of $A$ consists of the interval $\left[\lambda_{1}, \infty\right)$.

(iii) The derivative $(\mathrm{d} / \mathrm{d} \lambda)\left(P_{\lambda} f(\mathrm{x})\right)$ is given by (4.38). It is continuous on $\mathbb{R}$ if $l>2 m$. If $l \leqslant 2 m$, then $P_{\lambda} f$, in general, is not differentiable with respect to $\lambda$ at $\lambda=\lambda_{k}(k \in \mathbb{N})$.

\section{The principle of limiting amplitude}

We want to investigate the asymptotic behaviour of

$$
\begin{aligned}
u(\mathbf{x}, t)= & {\left[\int_{\lambda_{1}}^{\infty} \cos \sqrt{\lambda} t \mathrm{~d}\left(P_{\lambda} u_{0}\right)\right](\mathbf{x})+\left[\int_{\lambda_{1}}^{\infty} \frac{\sin \sqrt{\lambda} t}{\sqrt{\lambda}} \mathrm{d}\left(P_{\lambda} u_{1}\right)\right](\mathbf{x}) } \\
& +\left[\int_{\lambda_{1}}^{\infty} \psi(\lambda, t) \mathrm{d}\left(P_{\lambda} f\right)\right](\mathbf{x})
\end{aligned}
$$

as $t \rightarrow \infty$. Since $P_{\lambda}=0$ for $\lambda \leqslant \lambda_{1}, u$ coincides with the uniquely determined solution of (1.2)-(1.4) satisfying (2.12). We use the following lemma:

Lemma 5.1. Let $M$ be an arbitrary compact subset of $\bar{\Omega}$ and $s$ the smallest integer with $2(s+1)>n / 2$. Assume that $g \in C_{0}^{\infty}(\Omega)$. Then there exists a constant $c>0$ such that

$$
\left|\left[\int_{\alpha}^{\beta} \varphi(\lambda) \mathrm{d}\left(P_{\lambda} g\right)\right](\mathbf{x})\right| \leqslant c\left\|\left(P_{\beta}-P_{\alpha}\right) A^{s} g\right\| \sup _{\alpha<\lambda<\beta}|\varphi(\lambda)|
$$

(with $P_{\infty}:=I$ ) for every $\mathbf{x} \in M$, every interval $(\alpha, \beta)$ with $\lambda_{1} \leqslant \alpha<\beta \leqslant \infty$ and every bounded $\varphi \in C(\alpha, \beta)$.

The proof of Lemma 5.1 can be obtained by a modification of the proof of Lemma 3.1 in Reference 12.

Assume that $g \in C_{0}^{\infty}(\Omega)$ and that $\varphi$ is bounded and continuous in $(\alpha, \beta)$ with $\lambda_{1} \leqslant \alpha<\beta \leqslant \infty$. Lemma 5.1 implies

$$
\left[\int_{\alpha}^{\beta} \varphi(\lambda) \mathrm{d}\left(P_{\lambda} g\right)\right](\mathbf{x})=\int_{\alpha}^{\beta} \varphi(\lambda) \mathrm{d}\left(P_{\lambda} g(\mathbf{x})\right) \text {. }
$$

Since $\left\{P_{\lambda}\right\}$ is continuous for $\lambda \in \mathbb{R}$ and continuously differentiable with respect to $\lambda \in \mathbb{R} \backslash\left\{\lambda_{1}, \lambda_{2}, \ldots\right\}$, it follows from (4.39) that

$$
\int_{\alpha}^{\beta} \varphi(\lambda) \mathrm{d}\left(P_{\lambda} g(\mathbf{x})\right)=\int_{\alpha}^{\beta} \varphi(\lambda) \frac{\mathrm{d}\left(P_{\lambda} g(\mathbf{x})\right)}{\mathrm{d} \lambda} \mathrm{d} \lambda .
$$

Consider the first integral in (5.1),

$$
I_{1}(\mathbf{x}, t):=\int_{\lambda_{1}}^{\infty} \cos \sqrt{\lambda} t \mathrm{~d}\left(P_{\lambda} u_{0}(\mathbf{x})\right)
$$

In the remaining part of this section we consider a fixed compact subset $M$ of $\bar{\Omega}$ and a fixed $\varepsilon>0$. According to Lemma 5.1, there exists an $a>0$ such that

$$
I_{1}(\mathbf{x}, t)=\int_{\lambda_{1}}^{a} \cos \sqrt{\lambda} t \mathrm{~d}\left(P_{\lambda} u_{0}(\mathbf{x})\right)+w_{1}(\mathbf{x}, t)
$$

where $\left|w_{1}(\mathbf{x}, t)\right|<\varepsilon$ for $\mathbf{x} \in M$ and $t \geqslant 0$. The interval $\left[\lambda_{1}, a\right]$ contains only a finite number of eigenvalues $\lambda_{1}, \ldots \lambda_{s}$ of $(1.11)$. We set $U_{\delta}:=\bigcap_{j=i}^{s}\left(\lambda_{j}-\delta, \lambda_{j}+\delta\right) \cap\left[\lambda_{1}, a\right]$. 
Since $\left\{P_{\lambda}\right\}$ is continuous, Lemma 5.1 implies

$$
\left|\int_{U_{\mathrm{d}}} \cos \sqrt{\lambda} t \mathrm{~d}\left(P_{\lambda} u_{0}(\mathbf{x})\right)\right|<\varepsilon \quad \text { for } \mathbf{x} \in M
$$

for sufficiently small $\delta>0$. Note that the functions $(\mathrm{d} / \mathrm{d} \lambda)\left(P_{\lambda} u_{0}\right)$ and $\left(\mathrm{d}^{2} / \mathrm{d} \lambda^{2}\right)\left(P_{\lambda} u_{0}\right)$ are continuous and bounded for $\lambda \in\left[\lambda_{1}, a\right] \backslash U_{\delta}$ and $\mathbf{x} \in M$. Integrating by parts, we obtain

$$
\int_{\left[\lambda_{1}, a\right] \backslash U_{0}} \cos \sqrt{\lambda} t \frac{\mathrm{d}\left(P_{\lambda} u_{0}(\mathbf{x})\right)}{\mathrm{d} \lambda} \mathrm{d} \lambda=O\left(\frac{1}{t}\right) \quad \text { as } t \rightarrow \infty
$$

uniformly with respect to $\mathrm{x} \in M$. This, together with (5.4) and (5.6), yields $I_{1}(\mathrm{x}, t)$ $=o(1)$ as $t \rightarrow \infty$ uniformly in $M$.

The following lemma can be shown by analogous estimates:

Lemma 5.2. Let $M$ be a compact subset of $\bar{\Omega}$ and assume that $g \in C_{0}^{\infty}(\Omega)$. Then we have for every interval $(\alpha, \beta)$ with $\lambda_{1} \leqslant \alpha<\beta \leqslant \infty$ and every bounded $\varphi \in C(\alpha, \beta)$

$$
\begin{array}{ll}
\int_{\alpha}^{\beta} \varphi(\lambda) \cos \sqrt{\lambda} t \mathrm{~d}\left(P_{\lambda} g(x)\right)=o(1) & \text { as } t \rightarrow \infty, \\
\int_{\alpha}^{\beta} \varphi(\lambda) \sin \sqrt{\lambda} t \mathrm{~d}\left(P_{\lambda} g(x)\right)=o(1) & \text { as } t \rightarrow \infty,
\end{array}
$$

uniformly with respect to $\mathrm{x} \in M$.

We apply Lemma 5.2 to (5.1). Since

$$
\psi(\lambda, t)=\frac{\mathrm{e}^{-\mathrm{i} \omega t}-\mathrm{e}^{-\mathrm{i} \sqrt{\lambda} t}}{\lambda-\omega^{2}}-\frac{\mathrm{i} \sin \sqrt{\lambda} t}{\sqrt{\lambda}(\sqrt{\lambda}+\omega)}
$$

(compare (2.10), we obtain

$$
u(x, t)=\mathrm{e}^{-\mathrm{i} \omega t} \int_{\lambda_{1}}^{\infty} \frac{1-\mathrm{e}^{-\mathrm{i}(\sqrt{ } \lambda-\omega) t}}{\lambda-\omega^{2}} \mathrm{~d}\left(P_{\lambda} f(\mathbf{x})\right)+o(1) \quad \text { as } t \rightarrow \infty
$$

uniformly in $M$. For $\delta>0$ we set

$$
\begin{aligned}
I_{2}(\mathbf{x}, t ; \delta) & :=\int_{\omega^{2}-\delta}^{\omega^{2}+\delta} \frac{1-\mathrm{e}^{-i(\sqrt{\lambda}-\omega) t}}{\lambda-\omega^{2}} \mathrm{~d}\left(P_{\lambda} f(\mathbf{x})\right), \\
I_{3}(\mathbf{x} ; \delta) & :=\int_{\left|\lambda-\omega^{2}\right| \geqslant \delta} \frac{1}{\lambda-\omega^{2}} \mathrm{~d}\left(P_{\lambda} f(\mathbf{x})\right), \\
W_{1}(\mathbf{x}, t ; \delta): & =-\int_{\left|\lambda-\omega^{2}\right| \geqslant \delta} \frac{\mathrm{e}^{-i(\sqrt{\lambda}-\omega) t}}{\lambda-\omega^{2}} \mathrm{~d}\left(P_{\lambda} f(\mathbf{x})\right) .
\end{aligned}
$$

We denote by $W_{i}(i \in \mathbb{N})$ terms that will be shown later to be of order $o(1)$ as $t \rightarrow \infty$. By (5.9) $-(5.12)$ we have

$$
u(\mathbf{x}, t)=\mathrm{e}^{-\mathrm{i} \omega t}\left[I_{2}(\mathbf{x}, t ; \delta)+I_{3}(\mathbf{x} ; \delta)+W_{1}(\mathbf{x}, t ; \delta)\right]+o(1) \quad \text { as } t \rightarrow \infty,
$$

uniformly with respect to $\mathbf{x} \in M$ and $\delta>0$. 
Consider $I_{3}$ and note that

$$
\int_{\lambda_{1}}^{\infty} \frac{1}{\lambda-\omega^{2}-\mathrm{i} \tau} \mathrm{d}\left(P_{\lambda} f(\mathbf{x})\right)=R_{\omega^{2}+\mathrm{i} \tau} f(\mathbf{x})
$$

by the functional calculus for self-adjoint operators. Since

$$
\left|\frac{1}{\lambda-\omega^{2}}-\frac{1}{\lambda-\omega^{2}-i \tau}\right|=\frac{|\tau|}{\left|\lambda-\omega^{2}\right|\left|\lambda-\omega^{2}-i \tau\right|} \leqslant \frac{|\tau|}{\delta^{2}}
$$

for $\left|\lambda-\omega^{2}\right|>\delta$, we obtain by Lemma 5.1

$$
I_{3}(\mathbf{x} ; \delta)=\lim _{\tau \downarrow 0}\left[R_{\omega^{2}+\mathrm{i} \tau} f(\mathbf{x})-\int_{\omega^{2}-\delta}^{\omega^{2}+\delta} \frac{1}{\lambda-\omega^{2}-\mathrm{i} \tau} \mathrm{d}\left(P_{\lambda} f(\mathbf{x})\right)\right] .
$$

In the following we suppose that one of the conditions (4.23)-(4.25) holds with $\rho=\omega^{2}$. Then $R_{\omega^{2}+\mathrm{i} 0} f(\mathbf{x}):=\lim R_{\omega^{2}+\mathrm{i} \tau} f(\mathbf{x})$ exists. Furthermore, (4.38) and (4.39) imply that in this case $\lim (\mathrm{d} / \mathrm{d} \lambda)\left(P_{\lambda} f(\mathbf{x})\right)$ exists and that

$$
\left|\frac{\mathrm{d}\left(P_{\lambda} f(\mathbf{x})\right)}{\mathrm{d} \lambda}-\left[\frac{\mathrm{d}\left(P_{\lambda} f(\mathbf{x})\right)}{\mathrm{d} \lambda}\right]_{\lambda=\omega^{2}}\right|=O\left(\left|\dot{\lambda}-\omega^{2}\right|^{1 / 2 m}\right) \quad \text { as } \lambda \rightarrow \omega^{2}
$$

uniformly in $M$. Since

$$
\lim _{\tau \downarrow 0} \int_{\omega^{2}-\delta}^{\omega^{2}+\delta} \frac{1}{\lambda-\omega^{2}-\mathrm{i} \tau} \mathrm{d} \lambda=\mathrm{i} \pi
$$

we conclude from (5.15) that

$$
I_{3}(\mathbf{x} ; \delta)=R_{\omega^{2}+\mathrm{i} 0} f(\mathbf{x})-\mathrm{i} \pi\left[\frac{\mathrm{d}\left(P_{\lambda} f(\mathbf{x})\right)}{\mathrm{d} \lambda}\right]_{\lambda=\omega^{2}}+w_{2}(\mathbf{x} ; \delta),
$$

where $w_{2}(\mathbf{x} ; \delta) \rightarrow 0$ as $\delta \downarrow 0$ uniformly in $M$.

Now we turn to the discussion of $I_{2}$. As above we obtain

$$
I_{2}(\mathbf{x}, t ; \delta)=\int_{\omega^{2}-\delta}^{\omega^{2}+\delta} \frac{1-\mathrm{e}^{-\mathrm{i}(\sqrt{\lambda}-\omega) t}}{\lambda-\omega^{2}}\left[\frac{\mathrm{d}\left(P_{\lambda} f(\mathbf{x})\right)}{\mathrm{d} \lambda}\right]_{\lambda=\omega^{2}} \mathrm{~d} \lambda+w_{3}(\mathbf{x}, t ; \delta),
$$

where $w_{3}(\mathbf{x}, t ; \delta) \rightarrow 0$ as $\delta \downarrow 0$ uniformly in $M \times[0, \infty)$. It suffices to compute the integral in (5.18) for $\omega^{2} \geqslant \lambda_{1}$, since $\left[(\mathrm{d} / \mathrm{d} \lambda)\left(P_{\lambda} f(\mathbf{x})\right)\right]_{\lambda=\omega^{2}}=0$ for $\omega^{2}<\lambda_{1}$. We suppose that $0<\delta<\lambda_{1}$ and set $\mu:=\sqrt{ } \lambda, \xi:=\mu-\omega$. Then

$$
\begin{aligned}
\int_{\omega^{2}-\delta}^{\omega^{2}+\delta} \frac{1-\mathrm{e}^{-\mathrm{i}(\sqrt{ } \lambda-\omega) t}}{\lambda-\omega^{2}} \mathrm{~d} \lambda & =2 \int_{\sqrt{\left(\omega^{2}-\delta\right)}}^{\sqrt{\left(\omega^{2}+\delta\right)}} \frac{1-\mathrm{e}^{-\mathrm{i}(\mu-\omega) t}}{(\mu-\omega)(\mu+\omega)} \mu \mathrm{d} \mu \\
& =\int \sqrt{\sqrt{\left(\omega^{2}+\delta\right)}} \frac{1-\mathrm{e}^{-i(\mu-\omega) t}}{(\mu+\omega)} \mathrm{d} \mu+\int \sqrt{\sqrt{\left(\omega^{2}-\delta\right)}} \frac{\sqrt{\left(\omega^{2}-\delta\right)}-\omega}{\frac{1-\mathrm{e}^{-i \xi t}}{\xi}} \mathrm{d} \xi \\
& =\mathrm{i} \iint_{\sqrt{\left(\omega^{2}-\delta\right)}-\omega}^{\sqrt{\left(\omega^{2}+\delta\right)}-\omega} \frac{\sin \xi t}{\xi} \mathrm{d} \xi+w_{4}(t ; \delta)
\end{aligned}
$$


where

$$
w_{4}(t ; \xi)=\int_{\sqrt{\left(\omega^{2}-\delta\right)}}^{\sqrt{\left(\omega^{2}+\delta\right)}} \frac{1-\mathrm{e}^{-\mathrm{i}(\mu-\omega) t}}{(\mu+\omega)} \mathrm{d} \mu+\int_{\sqrt{\left(\omega^{2}-\delta\right)}-\omega}^{\sqrt{\left(\omega^{2}+\delta\right)}-\omega} \frac{1-\cos \xi t}{\xi} \mathrm{d} \xi .
$$

Note that $(1-\cos \xi t) / \xi$ is an odd function with respect to $\xi$. Therefore we have

$$
\begin{aligned}
& \left|\int_{\sqrt{\left(\omega^{2}-\delta\right)}-\omega}^{\sqrt{\left(\omega^{2}+\delta\right)}-\omega} \frac{1-\cos \xi t}{\xi} \mathrm{d} \xi\right|=\left|\int_{\omega-\sqrt{\left(\omega^{2}-\delta\right)}}^{\sqrt{\left(\omega^{2}+\delta\right)}-\omega} \frac{1-\cos \xi t}{\xi} \mathrm{d} \xi\right| \\
& \leqslant 2\left\{\ln \left[\sqrt{\left(1+\frac{\delta}{\omega^{2}}\right)}-1\right]-\ln \left[1-\sqrt{\left(1-\frac{\delta}{\omega^{2}}\right)}\right]\right\}=O(\delta) \quad \text { as } \delta \downarrow 0 .
\end{aligned}
$$

This implies $w_{4}(t ; \delta) \rightarrow 0$ as $\delta \downarrow 0$ uniformly in $[0, \infty)$. Setting $\zeta:=\xi t$, we obtain

$$
\int_{\sqrt{\left(\omega^{2}-\delta\right)}-\omega}^{\sqrt{\left(\omega^{2}+\delta\right)}-\omega} \frac{\sin \xi t}{\xi} \mathrm{d} \xi=\int_{\left[\sqrt{\left(\omega^{2}-\delta\right)}-\omega\right] t}^{\left[\sqrt{\left(\omega^{2}+\delta\right)}-\omega\right] t} \frac{\sin \zeta}{\zeta} \mathrm{d} \zeta=\pi+W_{2}(t ; \delta)
$$

with

$$
W_{2}(t ; \delta):=-\int_{\left[\sqrt{\left(\omega^{2}+\delta\right)}-\omega\right] t}^{\infty} \frac{\sin \zeta}{\zeta} \mathrm{d} \zeta-\int_{-\infty}^{\left[\sqrt{\left(\omega^{2}-\delta\right)}-\omega\right] \iota} \frac{\sin \zeta}{\zeta} \mathrm{d} \zeta,
$$

and hence

$$
\int_{\omega^{2}-\delta}^{\omega^{2}+\delta} \frac{1-\mathrm{e}^{-\mathrm{i}(\sqrt{\lambda}-\omega) t}}{\lambda-\omega^{2}} \mathrm{~d} \lambda=\mathrm{i} \pi+w_{4}(t ; \delta)+\mathrm{i} W_{2}(t ; \delta) .
$$

We conclude from (5.13), (5.17), (5.18) and (5.21) that

$$
u(\mathbf{x}, t)=\mathrm{e}^{-\mathrm{i} \omega t} R_{\omega^{2}+\mathrm{i} 0} f(\mathbf{x})+W_{3}(\mathbf{x}, t ; \delta)+w_{5}(\mathbf{x}, t ; \delta)+o(1)
$$

uniformly with respect to $\mathrm{x} \in M$, where

$$
W_{3}(\mathbf{x}, t ; \delta):=\mathrm{e}^{-\mathrm{i} \omega t}\left(W_{1}(x, t ; \delta)+\mathrm{i} W_{2}(t ; \delta)\left[\frac{\mathrm{d}\left(P_{\lambda} f(\mathbf{x})\right)}{\mathrm{d} \lambda}\right]_{\lambda=\omega^{2}}\right)
$$

and $w_{5}(\mathbf{x}, t ; \delta) \rightarrow 0$ as $\delta \downarrow 0$ uniformly with respect to $(\mathbf{x}, t) \in M \times[0, \infty)$. In particular, there exists a $\delta_{3}>0$ with $\left|w_{5}\left(\mathrm{x}, t ; \delta_{3}\right)\right|<\varepsilon$ for $\mathrm{x} \in M$ and $t \geqslant 0$. From (5.20), (5.12) and Lemma 5.2 we obtain $W_{3}\left(\mathbf{x}, t ; \delta_{3}\right)=o(1)$ as $t \rightarrow \infty$ uniformly in $M$. Altogether we have

$$
u(\mathbf{x}, t)=\mathrm{e}^{-\mathrm{i} \omega t} R_{\omega^{2}+\mathrm{i} 0} f(\mathbf{x})+o(1) \quad \text { as } t \rightarrow \infty
$$

uniformly in every bounded subset of $\bar{\Omega}$. This shows that the principle of limiting amplitude (1.8) holds if one of the conditions (4.23)-(4.25) is satisfied.

\section{Resonances}

In this section we investigate the solution $u$ under the assumptions $l \leqslant 2 m$ and $\omega^{2}=\lambda_{k}$. As above, we denote by $M$ a fixed compact subset of $\bar{\Omega}$.

We start our considerations from the asymptotic relation (5.13). First we consider the integral $I_{2}$ introduced in (5.10). Together with (5.4), (4.39), (4.40) and $\sigma=l / 2-1$, 
we obtain

$$
\begin{aligned}
I_{2}(\mathbf{x}, t ; \delta)= & \frac{1}{2 m(2 \pi)^{l / 2}} \sum_{s=0}^{[m-1 / 2]} C_{s} I_{\beta_{s}}^{*}(t ; \delta) p_{s}^{(k)}(\mathbf{x}) \\
& +\int_{\omega^{2}-\delta}^{\omega^{2}+\delta} \frac{1-\mathrm{e}^{-\mathrm{i}(\sqrt{\lambda}-\omega) t}}{\lambda-\omega^{2}} \mathrm{~d} \lambda g_{k}(\mathbf{x})+w_{6}(\mathbf{x}, t ; \delta),
\end{aligned}
$$

where

$$
\begin{aligned}
& \beta_{s}:=1-\frac{\sigma+1+s}{m}=1-\frac{l+2 s}{2 m}, \\
& I_{\beta}^{*}(t ; \delta):=\int_{\omega^{2}}^{\omega^{2}+\delta} \frac{1-\mathrm{e}^{-\mathrm{i}(\sqrt{\lambda}-\omega) t}}{\left(\lambda-\omega^{2}\right)^{1+\beta}} \mathrm{d} \lambda, \\
& p_{s}^{(k)}(\mathbf{x}):=\int_{\mathbf{R}^{\prime}} f^{(k)}\left(x^{\prime}, y\right)\left|x-x^{\prime}\right|^{2 s} \mathrm{~d} x^{\prime}
\end{aligned}
$$

and $w_{6}(\mathbf{x}, t ; \delta) \rightarrow 0$ as $\delta \downarrow 0$ uniformly in $M \times[0, \infty)$. Using (5.21), we conclude that

$$
\begin{aligned}
I_{2}(\mathbf{x}, t ; \delta)= & \frac{1}{2 m(2 \pi)^{l / 2}} \sum_{s=0}^{[m-1 / 2]} C_{s} I_{\beta_{s}}^{*}(t ; \delta) p_{s}^{(k)}(\mathbf{x})+\mathrm{i} \pi g_{k}(\mathbf{x}) \\
& +\mathrm{i} W_{2}(t ; \delta) g_{k}(\mathbf{x})+w_{7}(\mathbf{x}, t ; \delta)
\end{aligned}
$$

where $w_{7}(\mathbf{x}, t ; \delta) \rightarrow 0$ as $\delta \downarrow 0$ uniformly in $M \times[0, \infty)$.

Consider $I_{\beta}^{*}$. The substitution $\mu:=\sqrt{\lambda}$ yields

$$
I_{\beta}^{*}(t ; \delta)=2 \int_{\omega}^{\sqrt{\left(\omega^{2}+\delta\right)}} \frac{1-\mathrm{e}^{-\mathrm{i}(\mu-\omega) t}}{(\mu-\omega)^{i+\beta}} h_{\beta}(\mu) \mathrm{d} \mu,
$$

with $h_{\beta}(\mu):=\mu /(\mu+\omega)^{1+\beta}$. Note that

$$
\left|h_{\beta}(\mu)-h_{\beta}(\omega)\right| \leqslant|\mu-\omega|\left|\frac{\mathrm{d} h_{\beta}}{\mathrm{d} \mu}(\omega)\right|
$$

for $\mu \in[\omega, \omega / \beta]$. This implies

$$
I_{\beta}^{*}(t ; \delta)=\frac{1}{(2 \omega)^{\beta}} \int_{\omega}^{\sqrt{\left(\omega^{2}+\delta\right)}} \frac{1-\mathrm{e}^{-\mathrm{i}(\mu-\omega) t}}{(\mu-\omega)^{1+\beta}} \mathrm{d} \mu+w_{8}(t ; \delta ; \beta),
$$

where $w_{8}(t ; \delta ; \beta) \rightarrow 0$ as $\delta \downarrow 0$ uniformly with respect to $t \geqslant 0$ and $\beta \in[0,1-1 / 2 m]$. Setting $\xi:=(\mu-\omega) t$, we obtain

$$
I_{\beta}^{*}(t ; \delta)=\frac{t^{\beta}}{(2 \omega)^{\beta}} \int_{0}^{\left[\sqrt{\left(\omega^{2}+\delta\right)}-\omega\right] t} \frac{1-\mathrm{e}^{-\mathrm{i} \xi}}{\xi^{1+\beta}} \mathrm{d} \xi+w_{8}(t ; \delta ; \beta) .
$$


Assume that $0<\beta<1-1 / 2 m$. By (6.6) we have

$$
\begin{aligned}
I_{\beta}^{*}(t ; \delta) & =\frac{t^{\beta}}{(2 \omega)^{\beta}}\left[\int_{0}^{\infty} \frac{1-\mathrm{e}^{-\mathrm{i} \xi}}{\xi^{1+\beta}} \mathrm{d} \xi-\int_{\left[\sqrt{\left(\omega^{2}+\delta\right)}-\omega\right] t}^{\infty} \frac{\mathrm{d} \xi}{\xi^{1+\beta}}\right. \\
& \left.+\int_{\left[\sqrt{\left(\omega^{2}+\delta\right)}-\omega\right] t}^{\infty} \frac{\mathrm{e}^{-\mathrm{i} \xi}}{\xi^{1+\beta}} \mathrm{d} \xi\right]+w_{8}(t ; \delta ; \beta) \\
& =\frac{1}{(2 \omega)^{\beta}}\left[\frac{\pi \mathrm{e}^{\mathrm{i} \beta \pi / 2}}{\beta \Gamma(\beta) \sin (\beta \pi)} t^{\beta}-\frac{1}{\beta\left[\sqrt{\left(\omega^{2}+\delta\right)}-\omega\right]^{\beta}}\right] \\
& +w_{8}(t ; \delta ; \beta)+W_{4}(t ; \delta ; \beta),
\end{aligned}
$$

with

$$
W_{4}(t ; \delta ; \beta):=\frac{t^{\beta}}{(2 \omega)^{\beta}} \int_{\left[\sqrt{\left(\omega^{2}+\delta\right)}-\omega\right] t}^{\infty} \frac{\mathrm{e}^{-i \xi}}{\xi^{1+\beta}} \mathrm{d} \xi
$$

(compare the integrals (11c) and (12b) in Reference 1). Note that

$$
\left|W_{4}(t ; \delta ; \beta)\right| \leqslant \frac{2}{(2 \omega)^{\beta}\left[\sqrt{\left(\omega^{2}+\delta\right)}-\omega\right]^{1+\beta}} \frac{1}{t},
$$

as an integration by parts shows.

Now let $\beta=0$. In this case (6.6) can be rewritten as

$$
\begin{aligned}
I_{0}^{*}(t ; \delta)= & \int_{1}^{\left[\sqrt{\left(\omega^{2}+\delta\right)}-\omega\right] t} \frac{\mathrm{d} \xi}{\xi}+\int_{0}^{1} \frac{1-\mathrm{e}^{-\mathrm{i} \xi}}{\xi} \mathrm{d} \xi-\int_{1}^{\infty} \frac{\mathrm{e}^{-\mathrm{i} \xi}}{\xi} \mathrm{d} \xi \\
& +\int_{\left[\sqrt{\left(\omega^{2}+\delta\right)}-\omega\right] t}^{\infty} \frac{\mathrm{e}^{-\mathrm{i} \xi}}{\xi} \mathrm{d} \xi+w_{8}(t ; \delta ; 0) \\
= & \ln t+\ln \left[\sqrt{\left(\omega^{2}+\delta\right)}-\omega\right]+C_{\mathrm{e}}+\mathrm{i} \frac{\pi}{2}+w_{8}(t ; \delta ; 0)+W_{4}(t ; \delta ; 0)
\end{aligned}
$$

$\left(C_{\mathrm{e}}:=\right.$ the Euler-Mascheroni constant; compare (3.67) in Reference 11).

We insert (6.7) and (6.9) in (6.4) and use the abbreviations

$$
\begin{aligned}
& E_{s}:=\frac{C_{s}}{2 m(2 \pi)^{l / 2}} \frac{\pi \mathrm{e}^{i \beta_{s} \pi / 2}}{(2 \omega)^{\beta_{s}} \beta_{s} \Gamma\left(\beta_{s}\right) \sin \left(\beta_{s} \pi\right)}\left(s=0, \ldots,\left[m-\frac{l}{2}\right]\right), \\
& E^{*}:=\frac{1}{2 m(2 \pi)^{l / 2}} C_{m-(l / 2)}
\end{aligned}
$$

with $\beta_{s}=1-(l+2 s) / 2 m$ and $C_{s}$ defined by (4.10). Then we obtain for odd $l$

$$
\begin{aligned}
I_{2}(\mathbf{x}, t ; \delta)= & \sum_{s=0}^{m-(l+1) / 2} E_{s} t^{\beta_{s}} p_{s}^{(k)}(\mathbf{x})+i \pi g_{k}(\mathbf{x}) \\
& -\frac{1}{2 m(2 \pi)^{l / 2}} \sum_{s=0}^{m-(l+1) / 2} \frac{C_{s}}{(2 \omega)^{\beta_{s}} \beta_{s}\left[\sqrt{\left(\omega^{2}+\delta\right)}-\omega\right]^{\beta_{s}}} p_{s}^{(k)}(\mathbf{x}) \\
& +w_{9}(\mathbf{x}, t ; \delta)+W_{s}(\mathbf{x}, t ; \delta),
\end{aligned}
$$


and for even $l$

$$
\begin{aligned}
I_{2}(\mathbf{x}, t ; \delta)= & \sum_{s=0}^{m-1-l / 2} E_{s} t^{\beta_{s}} p_{s}^{(k)}(\mathbf{x})+E^{*} \ln t \cdot p_{m-(l / 2)}^{(k)}(\mathbf{x})+\mathrm{i} \pi g_{k}(\mathbf{x}) \\
& -\frac{1}{2 m(2 \pi)^{1 / 2}} \sum_{s=0}^{m-1-t / 2} \frac{C_{s}}{(2 \omega)^{\beta_{s}} \beta_{s}\left[\sqrt{\left(\omega^{2}+\delta\right)}-\omega\right]^{\beta_{s}}} p_{s}^{(k)}(\mathbf{x}) \\
& +\frac{C_{m-(l / 2)}}{2 m(2 \pi)^{l / 2}}\left\{\ln \left[\sqrt{\left(\omega^{2}+\delta\right)}-\omega\right]+C_{\mathrm{c}}+\frac{\mathrm{i} \pi}{2}\right\} p_{m-(l / 2)}^{(k)}(\mathbf{x}) \\
& +w_{9}(\mathbf{x}, t ; \delta)+W_{s}(\mathbf{x}, t ; \delta),
\end{aligned}
$$

where

$$
W_{s}(\mathbf{x}, t ; \delta):=\frac{1}{2 m(2 \pi)^{l / 2}} \sum_{s=0}^{[m-1 / 2]} C_{s} W_{4}\left(t ; \delta ; \beta_{s}\right) p_{s}^{(k)}(\mathbf{x})+\mathrm{i} W_{2}(t ; \delta) g_{k}(\mathbf{x})
$$

and $w_{9}(\mathbf{x}, t ; \delta) \rightarrow 0$ as $\delta \downarrow 0$ uniformly in $M \times[0, \infty)$.

Now we turn to the discussion of $I_{3}$, using the respresentation (5.15). We conclude from (4.39) that

$$
\begin{aligned}
\int_{\omega^{2}-\delta}^{\omega^{2}+\delta} \frac{1}{\lambda-\omega^{2}-\mathrm{i} \tau} \frac{\mathrm{d} P_{\lambda} f(\mathbf{x})}{\mathrm{d} \lambda} \mathrm{d} \lambda= & \frac{1}{2 m(2 \pi)^{1 / 2}} \sum_{s=0}^{[m-l / 2]} C_{s} J_{\beta_{s}}^{*}(\delta ; \tau) p_{s}^{(k)}(\mathbf{x}) \\
& +\int_{\omega^{2}-\delta}^{\omega^{2}+\delta} \frac{1}{\lambda-\omega^{2}-\mathrm{i} \tau} \mathrm{d} \lambda g_{k}(\mathbf{x})+w_{10}(\mathbf{x} ; \delta ; \tau),
\end{aligned}
$$

where

$$
J_{\beta}^{*}(\delta ; \tau):=\int_{\omega^{2}}^{\omega^{2}+\delta} \frac{1}{\left(\lambda-\omega^{2}-\mathrm{i} \tau\right)\left(\lambda-\omega^{2}\right)^{\beta}} \mathrm{d} \lambda
$$

and $w_{10}(\mathbf{x} ; \delta ; \tau) \rightarrow 0$ as $\delta \downarrow 0$ uniformly with respect to $\mathbf{x} \in M$ and $\tau \geqslant 0$.

Consider $J_{\beta}^{*}$. Setting $\mu:=\left(\lambda-\omega^{2}\right) / \tau$, we obtain in the case $\beta>0$

$$
\begin{aligned}
J_{\beta}^{*}(\delta ; \tau) & =\frac{1}{\tau^{\beta}} \int_{0}^{\delta / \tau} \frac{\mathrm{d} \mu}{(\mu-\mathrm{i}) \mu^{\beta}} \\
& =\frac{1}{\tau^{\beta}}\left\{\int_{0}^{\infty} \frac{\mathrm{d} \mu}{(\mu-\mathrm{i}) \mu^{\beta}}-\int_{\delta / \tau}^{\infty}\left(\frac{1}{\mu^{\beta+1}}+\frac{\mathrm{i}}{(\mu-\mathrm{i}) \mu^{\beta+1}}\right) \mathrm{d} \mu\right\}, \\
& =\frac{1}{\tau^{\beta}} C^{*}(\beta)-\frac{1}{\beta \delta^{\beta}}+O(\tau) \quad \text { as } \tau \downarrow 0,
\end{aligned}
$$

with

$$
\begin{aligned}
C^{*}(\beta):= & \int_{0}^{\infty} \frac{\mathrm{d} \mu}{(\mu-\mathrm{i}) \mu^{\beta}}=\int_{0}^{\infty} \frac{\mathrm{d} \mu}{\left(\mu^{2}+1\right) \mu^{\beta-1}}+\mathrm{i} \int_{0}^{\infty} \frac{\mathrm{d} \mu}{\left(\mu^{2}+1\right) \mu^{\beta}} \\
& =\frac{\pi}{2}\left(\frac{1}{\sin \beta \pi / 2}+\frac{\mathrm{i}}{\sin (\beta+1) \pi / 2}\right)
\end{aligned}
$$

(compare the integral 42 on page 70 in Reference 1). In the case $\beta=0$ we have

$$
J_{0}^{*}(\delta ; \tau)=\ln \delta-\ln \tau+\frac{\mathrm{i} \pi}{2}+o(1) \quad \text { as } \tau \downarrow 0 .
$$


Together with (5.16) we obtain from (6.14)

$$
\begin{aligned}
\int_{\omega^{2}-\delta}^{\omega^{2}+\delta} \frac{1}{\lambda-\omega^{2}-\mathrm{i} \tau} \mathrm{d}\left(P_{\lambda} f(x)\right) & =\frac{1}{2 m(2 \pi)^{1 / 2}} \sum_{s=0}^{m-(l+1) / 2} C_{s}\left(\frac{C^{*}\left(\beta_{s}\right)}{\tau^{\beta_{s}}}-\frac{1}{\beta_{s} \delta^{\beta_{s}}}\right) p_{s}^{(k)}(\mathbf{x}) \\
& +\mathrm{i} \pi g_{k}(\mathbf{x})+w_{10}(\mathbf{x}, t ; \delta)+o(1) \quad \text { as } \tau \downarrow 0 \quad(6.19)
\end{aligned}
$$

if $l$ is odd, and

$$
\begin{aligned}
\int_{\omega^{2}-\delta}^{\omega^{2}+\delta} \frac{1}{\lambda-\omega^{2}-\mathrm{i} \tau} \mathrm{d}\left(P_{\lambda} f(\mathbf{x})\right)= & \frac{1}{2 m(2 \pi)^{l / 2}} \sum_{s=0}^{m-1-l / 2} C_{s}\left(\frac{C^{*}\left(\beta_{s}\right)}{\tau^{\beta_{s}}}-\frac{1-}{\beta_{s} \delta^{\beta_{s}}}\right) p_{s}^{(k)}(\mathbf{x}) \\
& +\frac{C_{m}-(l / 2)}{2 m(2 \pi)^{l / 2}}\left(\ln \delta-\ln \tau+\frac{\mathrm{i} \pi}{2}\right) p_{m-(l i 2)}^{(k)}(\mathbf{x}) \\
& +\mathrm{i} \pi g_{k}(\mathbf{x})+w_{10}(\mathbf{x} ; \delta ; \tau)+o(1) \quad \text { as } \tau \downarrow 0
\end{aligned}
$$

if $l$ is even.

Now we investigate the first term in (5.15). By Lemma 3.1 and (4.34) we have

$$
R_{\omega^{2}+\mathrm{i \tau}} f(\mathrm{x})=\sum_{j=1}^{\infty} u^{(j)}\left(\mathrm{x} ; \omega^{2}+\mathrm{i} \tau\right)
$$

The discussion in Sections 3 and 4 yields

$$
\sum_{\substack{j=1 \\ j \neq k}}^{\infty} u^{(j)}\left(\mathbf{x} ; \omega^{2}+\mathrm{i} \tau\right)=\sum_{\substack{j=1 \\ j \neq k}}^{\infty} u^{(j)}\left(\mathbf{x} ; \omega^{2}+\mathrm{i} 0\right)+o(1) \quad \text { as } \tau \downarrow 0,
$$

uniformly in $M$. From (4.15), (4.16) and (6.3) we obtain

$$
u^{(k)}\left(\mathbf{x} ; \omega^{2}+\mathrm{i} \tau\right)=v^{(k)}(\mathbf{x})+\sum_{s=0}^{m-(l+1) / 2} \frac{C_{s} D_{s}}{\tau^{\beta_{s}}} p_{s}^{(k)}(\mathbf{x})+O\left(\tau^{1 / 2 m}\right) \quad \text { as } \tau \downarrow 0
$$

for odd $l$, and

$$
\begin{aligned}
u^{(k)}\left(\mathbf{x} ; \omega^{2}+\mathrm{i} \tau\right)= & v^{(k)}(\mathbf{x})-D^{\prime}(1) p_{m}^{(k)}-(l / 2) \\
& +\sum_{s=0}^{m-1} \frac{(\mathbf{x})-\frac{C_{m}-(l / 2)}{2 m(2 \pi)^{l / 2}} \ln \tau \cdot p_{m}^{(k)}-(l / 2)}{\tau_{s}^{\beta_{s}}} p_{s}^{(\mathbf{k})}(\mathbf{x})+O\left(\tau^{1 / 2 m}\right) \quad \text { as } \tau \downarrow 0
\end{aligned}
$$

for even $l$, where

$$
v^{(k)}(\mathbf{x}):= \begin{cases}\frac{\Gamma((l / 2)-m)}{\pi^{l / 2} 4^{m}(m-1) !} & \text { if } l \text { is odd } \\ \times \int_{\mathbb{R}^{\prime}} f^{(k)}\left(x^{\prime}, y\right)\left|x-x^{\prime}\right|^{2 m-1} \mathrm{~d} x^{\prime} & \\ \frac{(-1)^{m+1-(l / 2)}}{\pi^{l / 2} 2^{2 m-1}(m-(l / 2)) !(m-1) !} & \\ \times \int_{\mathbf{R}^{\prime}} f^{(k}\left(x^{\prime}, y\right)\left|x-x^{\prime}\right|^{2 m-l} \ln \left|x-x^{\prime}\right| \mathrm{d} x^{\prime} & \text { if } l \text { is even }\end{cases}
$$


We set

$$
V_{\omega}(\mathrm{x}):= \begin{cases}\sum_{\substack{j=1 \\ j \neq k}}^{\infty} u^{(j)}\left(\mathrm{x} ; \omega^{2}+\mathrm{i} 0\right)+v^{(k)}(\mathrm{x}) & \text { for odd } l \\ \left.\left(\frac{C_{m-(l / 2)}}{2 m(2 \pi)^{l / 2}} \mid C_{e}-\ln (2 \omega)\right]-D^{\prime}(1)\right) p_{m-(l / 2)}^{(k)}(\mathbf{x}) & \\ +\sum_{\substack{j=1 \\ j \neq k}}^{\infty} u^{(j)}\left(\mathbf{x} ; \omega^{2}+\mathrm{i} 0\right)+v^{(k)}(\mathbf{x}) & \text { for even } l .\end{cases}
$$

Note that $\left[\left(-\Delta_{x}\right)^{m}+\left(-\Delta_{y}\right)^{m}\right] p_{m-(l / 2)}^{(k)}(\mathbf{x})-\omega^{2} p_{m-(1 / 2)}^{(k)}(\mathbf{x})=0$ by $(6.3)$ and (4.34) and that therefore

$$
\begin{array}{ll}
{\left[\left(-\Delta_{x}\right)^{m}+\left(-\Delta_{y}\right)^{m}\right] V_{\omega}-\omega^{2} V_{\omega}=f} & \text { in } \Omega, \\
V_{\omega}=\frac{\partial V_{\omega}}{\partial \mathbf{n}}=\ldots=\frac{\partial^{m-1} V_{\omega}}{\partial \mathbf{n}^{m-1}} & \text { on } \partial \Omega .
\end{array}
$$

Now we assume that $l$ is odd. Recall that $\beta_{s}=1-(l+2 s) / 2 m$ and $\sigma=(l / 2)-1$. Note that by (4.12) and (6.17)

$$
C_{s} D_{s}=\frac{C_{s} C^{*}\left(\beta_{s}\right)}{2 m(2 \pi)^{1 / 2}} \quad\left(s=0,1, \ldots, m-\frac{l+1}{2}\right)
$$

if $\tau>0$. Therefore (5.15), (6.19) and (6.21)-(6.26) yield

$$
l_{3}(\mathbf{x} ; \delta)=V_{\omega}(\mathbf{x})+\frac{1}{2 m(2 \pi)^{l / 2}} \sum_{s=0}^{m-(l+1) / 2} \frac{C_{s}}{\beta_{s} \delta^{\beta_{s}}} p_{s}^{(k)}(\mathbf{x})-\mathrm{i} \pi g_{k}(\mathbf{x})-w_{10}(\mathbf{x} ; \delta ; 0) .
$$

Combining this with (6.11), we obtain

$$
\begin{aligned}
I_{2}(\mathbf{x}, t ; \delta)+I_{3}(\mathbf{x} ; \delta)= & \sum_{s=0}^{m-(l+1) / 2} E_{s} t^{\beta_{s}} p_{s}^{(k)}(\mathbf{x})+V_{\omega}(\mathbf{x}) \\
& +\sum_{s=0}^{m-(l+1) / 2} \frac{1}{2 m(2 \pi)^{l / 2}} C_{s} \\
& \times\left(\frac{1}{\beta_{s} \delta^{\beta_{s}}}-\frac{1}{\left.(2 \omega)^{\beta_{s}} \beta_{s}\left[\sqrt{\left(\omega^{2}+\delta\right)}-\omega\right)-\omega\right]^{\beta_{s}}}\right) p_{s}^{(k)}(\mathbf{x}) \\
& +w_{11}(\mathbf{x}, t ; \delta)+W_{s}(\mathbf{x}, t ; \delta),
\end{aligned}
$$

where $w_{11}(\mathbf{x}, t ; \delta) \rightarrow 0$ as $\delta \downarrow 0$ uniformly in $M \times[0, \infty)$. Note that

$$
\begin{aligned}
\lim _{\delta \downarrow 0}\left[\frac{1}{\beta \delta^{\beta}}-\frac{1}{(2 \omega)^{\beta} \beta\left[\sqrt{\left(\omega^{2}+\delta\right)}-\omega\right]^{\beta}}\right] & =\frac{1}{\beta} \lim _{\delta \downarrow 0} \frac{(2 \omega)^{\beta}-\left[\sqrt{\left(\omega^{2}+\delta\right)}+\omega\right]^{\beta}}{(2 \omega)^{\beta} \delta^{\beta}} \\
& =-\frac{1}{2 \beta(2 \omega)^{\beta}} \lim _{\delta \downarrow 0} \frac{\left[\sqrt{\left(\omega^{2}+\delta\right)}+\omega\right]^{\beta-1}}{\delta^{\beta-1} \sqrt{\left(\omega^{2}+\delta\right)}} \\
& =0
\end{aligned}
$$


for $0<\beta<1$. Hence, for any given $\varepsilon>0$ there exists a $\delta_{4}>0$ such that

$$
\left|\frac{1}{2 m(2 \pi)^{1 / 2}} \sum_{s=0}^{m-(l+1) / 2} C_{s}\left(\frac{1}{\beta_{s} \delta_{4}^{\beta_{s}}}-\frac{1}{(2 \omega)^{\beta_{s}} \beta_{s}\left[\sqrt{\left(\omega^{2}+\delta_{4}\right)}-\omega\right]^{\beta_{z}}}\right) p_{s}^{(k)}(\mathbf{x})\right|<\varepsilon
$$

and $\left|w_{11}\left(\mathbf{x}, t ; \delta_{4}\right)\right|<\varepsilon$ for $\mathbf{x} \in M$. We insert (6.29) into (5.13). Note that by (5.12), Lemma $5.2,(6.13),(5.20)$ and $(6.8)$

$$
W_{5}\left(\mathbf{x}, t ; \delta_{4}\right)+W_{1}\left(\mathbf{x}, t ; \delta_{4}\right)=o(1) \quad \text { as } t \rightarrow \infty \text {. }
$$

uniformly with respect to $\mathrm{x} \in M$. Thus we obtain

$$
u(\mathbf{x}, t)=\sum_{s=0}^{m-(l+1) / 2} E_{s} \mathrm{e}^{-\mathrm{i} \omega t} t^{\beta_{s}} p_{s}^{(k)}(\mathbf{x})+\mathrm{e}^{-\mathrm{i} \omega t} V_{\omega}(\mathbf{x})+o(1) \quad \text { as } t \rightarrow \infty
$$

uniformly with respect to $\mathrm{x} \in M$ if $l$ is odd.

Now we assume that $l$ is even. As above we conclude from (5.15), (6.20) and (6.21)-(6.26)

$$
\begin{aligned}
I_{3}(\mathbf{x} ; \delta)= & V_{\omega}(\mathbf{x})+\frac{C_{m-(l / 2)}}{2 m(2 \pi)^{1 / 2}}\left(\ln 2 \omega-C_{e}\right) p_{m-(l / 2)}^{(k)}(\mathbf{x})-\mathrm{i} \pi g_{k}(\mathbf{x}) \\
& +\frac{1}{2 m(2 \pi)^{1 / 2}} \sum_{s=0}^{m-1-(l / 2)} \frac{C_{s}}{\beta_{s} \delta^{\beta_{s}}} p_{s}^{(k)}(\mathbf{x}) \\
& -\frac{C_{m-(l / 2)}}{2 m(2 \pi)^{l / 2}}\left(\ln \delta+\frac{\mathrm{i} \pi}{2}\right) p_{m-(l i 2)}^{(k)}(\mathbf{x})-w_{10}(\mathbf{x} ; \delta ; 0)
\end{aligned}
$$

and with (6.12)

$$
\begin{aligned}
I_{2}(\mathbf{x}, t ; \delta)+ & I_{3}(\mathbf{x} ; \delta) \\
= & \sum_{s=0}^{m-1-(l / 2)} E_{s} t^{\beta_{s}} p_{s}^{(k)}(\mathbf{x})+E^{*} \ln t \cdot p_{m-(l / 2)}^{(\mathbf{k})}(\mathbf{x})+V_{\omega}(\mathbf{x}) \\
& +\frac{C_{m-(l / 2)}}{2 m(2 \pi)^{l / 2}}\left\{\ln 2 \omega+\ln \left[\sqrt{\left(\omega^{2}+\delta\right)}-\omega\right]-\ln \delta\right\} p_{m-(l / 2)}^{(k)}(\mathbf{x}) \\
& +w_{12}(\mathbf{x}, t ; \delta)+W_{5}(\mathbf{x}, t ; \delta),
\end{aligned}
$$

where $w_{12}(\mathbf{x}, t ; \delta) \rightarrow 0$ as $\delta \downarrow 0$ uniformly with respect to $\mathbf{x} \in M$ and $t \geqslant 0$. Since

$$
\lim _{\delta \downarrow 0}\left\{\ln \left[\sqrt{\left(\omega^{2}+\delta\right)}-\omega\right]-\ln \delta\right\}=-\ln 2 \omega .
$$

we obtain together with (5.13)

$$
\begin{aligned}
u(\mathbf{x}, t)= & \sum_{s=0}^{m-1-(l / 2)} E_{s} \mathrm{e}^{-i \omega t} t^{\beta_{s}} p_{s}^{(k)}(\mathbf{x})+E^{*} \mathrm{e}^{-i \omega t} \ln t \cdot p_{m-(l / 2)}^{(k)}(\mathbf{x}) \\
& +\mathrm{e}^{-i \omega t} V_{\omega}(\mathbf{x})+o(1) \quad \text { as } t+\infty
\end{aligned}
$$

uniformly in $M$. Thus we have proved:

Theorem 6.1. Let $u$ be the uniquely determined solution of (1.2)-(1.4) satisfying (2.12) and assume that $f, u_{1}, u_{2} \in C_{0}^{\infty}(\Omega), \partial \Omega \in C^{\infty}$ and $\Omega=\Omega^{\prime} \times \mathbb{R}^{l}$. The principle of limiting amplitude ((1.8) and (1.10)) holds if and only if one of the conditions (4.23)-(4.25) is 
satisfied. The limit amplitude $U_{\omega}$ coincides with the limit $R_{\omega^{2}+i 0}$ fof the resolvent of the operator $A$ introduced in (2.1) and is uniquely characterized by the conditions stated in Lemma 4.1 .

In all other cases resonances of order $t^{\alpha}(0<\alpha<1)$ or lnt occur. The asymptotic behaviour of $u$ in the resonance case is given by (6.30) ifl is odd and by (6.33) if l is even (with $\left.\beta_{s}=1-(l+2 s) / 2 m\right)$.

\section{References}

1. Bronstein, 1. N. and Semendjajew, K. A., Taschenbuch der Mathematik, Harri Deutsch, Thun, 1984.

2. Eidus, D. M., 'The principle of limit amplitude', Uspekhi Mat. Nauk, 24, (1969). English transl.: Russian Math. Surveys, 24, 97-167 (1969).

3. Lesky, P., 'Resonanzphänomene in Wellenleitern für eine Klasse partieller Differentialgleichungen höherer Ordnung', Thesis, University of Stuttgart, 1987.

4. Lesky, P., 'A uniqueness condition for the polyharmonic equation in free space', to appear.

5. Magnus, W. and Oberhettinger, F., Formeln und Sätze für die speziellen Funktionen der mathematischen Physik, Springer, Berlin, 1948.

6. Ramm, A. G. and Werner, P., 'On the limit amplitude principle for a layer', J. Reine Angew. Math., 360, $19-46$ (1985).

7. Sveshnikov, A. G., 'The limit absorption principle for a waveguide', Dokl. Akad. Nauk SSSR, 80, 341-344 (1951).

8. Werner, P., 'A distribution-theoretical approach to certain Lebesgue and Sobolev spaces', J. Math. Anal. Appl., 29, 18-78 (1970).

9. Werner, P., 'Self-adjoint extensions of the Laplace operator with respect to electric and magnetic boundary conditions', J. Math. Anal. Appl., 70, 131-160 (1979).

10. Werner, P., 'Regularity properties of the Laplace operator with respect to electric and magnetic boundary conditions', J. Math. Anal. Appl., 87, 560-602 (1982).

11. Werner, P., 'Zur Asymptotik der Wellengleichung und der Wärmeleitungsgleichung in zweidimensionalen Außenräumen', Math. Meth. in the Appl. Sci., 7, 170-201 (1985).

12. Werner, P., 'Resonance phenomena in cylindrical waveguides'; J. Math. Anal. Appl., 121, 173-214 (1987). 